\title{
The Grossmann-Royer transform, Gelfand-Shilov spaces, and continuity properties of localization operators on modulation spaces
}

Nenad Teofanov

\begin{abstract}
This paper offers a review of the results concerning localization operators on modulation spaces, and related topics. However, our approach, based on the Grossmann-Royer transform, gives a new insight and (slightly) different proofs. We define the Grossmann-Royer transform as interpretation of the Grossmann-Royer operator in the weak sense. Although such transform is essentially the same as the cross-Wigner distribution, the proofs of several known results are simplified when it is used instead of other time-frequency representations. Due to the importance of their role in applications when dealing with ultrafast decay properties in phase space, we give a detailed account on the Gelfand-Shilov spaces and their dual spaces, and extend the Grossmann-Royer transform and its properties in such context. Another family of spaces, modulation spaces, are recognized as appropriate background for time-frequency analysis. In particular, the Gelfand-Shilov spaces are revealed as projective and inductive limits of modulation spaces. For the continuity and compactness properties of localization operators we employ the norms in modulation spaces. We define localization operators in terms of the GrossmannRoyer transform, and show that such definition coincides with the usual definition based on the short-time Fourier transform.
\end{abstract}

\section{Introduction}

The Grossmann-Royer operators originate from the problem of physical interpretation of the Wigner distribution, see [44, 62]. It is shown that the Wigner distribution is related to the expectation values of such operators which describe reflections about the phase space point. Apart from this physically plausible interpretation of the Grossmann-Royer operators (sometimes called the parity operators),

Nenad Teofanov

Department of Mathematics and Informatics, University of Novi Sad, Novi Sad, Serbia, e-mail: nenad.teofanov@dmi.uns.ac.rs 
they are closely related to the Heisenberg operators, the well known objects from quantum mechanics as "quantized" variants of phase space translations. We refer to $[23,24,25]$ for such relation, basic properties of those operators, and the definition of the cross-Wigner distribution $W(f, g)$ in terms of the Grossmann-Royer operator $R f$.

As it is observed in de Gosson [23] it is a pity that the Grossmann-Royer operators are not universally known. One of the aims of this paper is to promote those operators by defining the Grossmann-Royer transforms $R_{g} f$ as their weak interpretation. The Grossmann-Royer transform is in fact the cross-Wigner distribution multiplied by $2^{d}$, see Definition 1, Lemma 1 and [25, Definition 12]. Thus all the results valid for $W(f, g)$ can be formulated in terms of $R_{g} f$. Nevertheless, due to its physical meaning, we treat the Grossmann-Royer transform as one of the timefrequency representations and perform a detailed exposition of its properties. Our calculations confirm the remark from [23, Chapter 8.3] accorindg to which the use of $R_{g} f$ (instead of $V_{g} f, W(f, g)$ and $A(f, g)$, see Definition 1) "allows one to considerably simplify many proofs".

One aim of this paper is to give the interpretation of localization operators by the means of the Grossmann-Royer transforms, and to reveal its role in time-frequency analysis, but we believe that, due to its intrinsic physical interpretation (as reflections in phase space), the study of the Grossmann-Royer transform is of an interest by itself.

To that end, we extend the definition of the Grossmann-Royer transform from the duality between the Schwartz space $\mathscr{S}\left(\mathbb{R}^{d}\right)$ and its dual space of tempered distributions $\mathscr{S}^{\prime}\left(\mathbb{R}^{d}\right)$ to the whole range of Gelfand-Shilov spaces and their dual spaces. According to [52], such spaces are "better adapted to the study of the problems of Applied Mathematics", since they describe simultaneous estimates of exponential type for a function and its Fourier transform. Here we give a novel (albeit expected) characterization of Gelfand-Shilov spaces in terms of the Grossmann-Royer transform.

Gelfand-Shilov spaces can be described as the projective and the inductive limits of modulation spaces, which are recognized as the right spaces for time-frequency analysis [38]. The modulation space norms traditionally measure the joint timefrequency distribution of $f \in \mathscr{S}^{\prime}$, since their weighted versions are usually equipped with weights of at most polynomial growth at infinity ([29], [38, Ch. 11-13]). The general approach to modulation spaces introduced already in [29] includes the weights of sub-exponential growth (see (19)). However, the study of ultradistributions requires the use of the weights of exponential or even superexponential growth, cf. $[19,80]$. In our investigation of localization operators it is essential to use the (sharp) convolution estimates for modulation spaces, see Section 4.

Localization operators are closely related to the above mentioned cross-Wigner distribution. They were first introduced by Berezin in the study of general Hamiltonians satisfying the so-called Feynman inequality, within a quantization problem in quantum mechanics, [5, 64]. Such operators and their modifications are also called Toeplitz or Berezin-Toeplitz operators, anti-Wick operators and Gabor multipliers, 
see $[34,77,78]$. We do not intend to discuss the terminology here, and refer to, e.g. [27] for the relation between Toeplitz operators and localization operators.

In signal analysis, different localization techniques are used to describe signals which are as concentrated as possible in general regions of the phase space. In particular, localization operators are related to localization technique developed by Slepian-Polak-Landau, where time and frequency are considered to be two separate spaces, see e.g. the survey article [67]. A different construction is proposed by Daubechies in [22], where time-frequency plane is treated as one geometric whole (phase space). That fundamental contribution which contains localization in phase space together with basic facts on localization operators with references to applications in optics and signal analysis, initiated further study of the topic. More precisely, Daubechies studied localization operators $A_{a}^{\varphi_{1}, \varphi_{2}}$ with Gaussian windows

$\varphi_{1}(x)=\varphi_{2}(x)=\pi^{-d / 4} \exp \left(-x^{2} / 2\right), x \in \mathbb{R}^{d}$, and with a radial symbol $a \in L^{1}\left(\mathbb{R}^{2 d}\right)$.

Such operators are named Daubechies' operators afterwards. The eigenfunctions of Daubechies' operators are $d$-dimensional Hermite functions

$$
H_{k}(x)=H_{k_{1}}\left(x_{1}\right) H_{k_{2}}\left(x_{2}\right) \ldots H_{k_{d}}\left(x_{d}\right)=\prod_{j=1}^{d} H_{k_{j}}\left(x_{j}\right), \quad x \in \mathbb{R}^{d}, \quad k \in \mathbb{N}_{0}^{d},
$$

and

$$
H_{n}(t)=(-1)^{n} \pi^{-1 / 4}\left(2^{n} n !\right)^{-1 / 2} \exp \left(t^{2} / 2\right)\left(\exp \left(-t^{2}\right)\right)^{(n)}, \quad t \in \mathbb{R}, \quad n=0,1, \ldots,
$$

and corresponding eigenvalues can be explicitly calculated. Inverse problem for a simply connected localization domain $\Omega$ is studied in [1] where it is proved that if one of the eigenfunctions of Daubechies' operator is a Hermite function, then $\Omega$ is a disc centered at the origin. Moreover, the Hermite functions belong to test function spaces for ultra-distributions, both in non-quasianalytic and in quasianalytic case, and give rise to important representation theorems, [47]. In our analysis this fact is used in Theorem 2.

The eigenvalues and eigenfunctions in signal analysis are discussed in [60], where the localization operators of the form $\left\langle L_{\chi_{\Omega}} f, g\right\rangle=\iint_{\Omega} W(f, g)$, were observed. Here $\Omega$ is a quite general open and bounded subset in $\mathbb{R}^{2}, \chi_{\Omega}$ is its characteristic function, and $W(f, g)$ is the cross-Wigner transform (see Definition 1). In [60] it is proved that the eigenfunctions of $L_{\chi_{\Omega}}$ belong to the Gelfand-Shilov space $\mathscr{S}^{(1)}\left(\mathbb{R}^{d}\right)$, see Section 3 for the definition.

Apart from applications in signal analysis, the study of localization operators is motivated (and in fact initiated by Berezin) by the problem of quantization. An exposition of different quantization theories and connection between localization operators and Toeplitz operators is given in e.g. [27, 28]. The quantization problem is in the background of the study of localization operators on $L^{p}(G), 1 \leq p \leq \infty$, where $G$ is a locally compact group, see [7,89], where one can find product formulae and Schatten-von Neumann properties of localization operators in that context. 
In the context of time-frequency analysis, the groundbreaking contribution is given by Cordero and Gröchenig, [13]. Among other things, their results emphasize the role of modulation spaces in the study of localization operators. Since then, localization operators in the context of modulation spaces and Wiener-amalgam spaces were studied by many authors, cf. [32, 14, 76, 77, 87]. See also the references given there.

In this paper we rewrite the definition of localization operators and Weyl pseudodifferential operators in terms of the Grossmann-Royer transform and recover the main relations between them. Thereafter we give an exposition of results concerning the continuity of localization operators on modulation spaces with polynomial weights, and their compactness properties both for polynomial and exponential weights.

\subsection{The content of the paper}

This paper is mainly a review of the results on the topic of localization operators on modulation spaces with some new insights and (slightly) different proofs. More precisely, we propose a novel approach based on the Grossmann-Royer transform $R_{g} f$, defined in Section 2 as the weak interpretation of the Grossmann-Royer operator. Therefore $R_{g} f$ is the cross-Wigner distribution in disguise (Definition 1 and [25, Definition 12]), so all the results valid for $W(f, g)$ can be reformulated in terms of $R_{g} f$. In fact, $R_{g} f$ is also closely related to other time-frequency representations, $V_{g} f$ and $A(f, g)$, see Lemma 1 . We perform a detailed exposition of the properties of the Grossmann-Royer transform, such as marginal densities and the weak uncertainty principle, Lemma 2 and Proposition 3, respectively. The relation between the Grossmann-Royer transform and the Heisenberg-Weyl (displacement) operators via the symplectic Fourier transform is revealed in Proposition 5.

We start Section 3 with a motivation for the study of Gelfand-Shilov spaces, and proceed with a review of their main properties, including their different characterizations (Theorem 1), the kernel theorem (Theorem 2), and a novel (albeit expected) characterization in terms of the Grossmann-Royer transform (Theorem 3).

In Section 4 we first discuss weight functions, and then define modulation spaces as a subset of $\left(\mathscr{S}_{1 / 2}^{1 / 2}\right)^{\prime}\left(\mathbb{R}^{d}\right)$, the dual space of the Gelfand-Shilov space $\mathscr{S}_{1 / 2}^{1 / 2}\left(\mathbb{R}^{d}\right)$, see Definition 5. This section is an exposition of known results relevant for the rest of the paper. In particular, we recall that Gelfand-Shilov spaces can be described as the projective and the inductive limits of modulation spaces, Theorem 4 and Remark 6. We also recall the (sharp) convolution estimates for modulation spaces (Proposition 7 and Theorem 5) which will be used in the study of localization operators in Section 5 .

In Section 5 we rewrite the definition of localization operators and Weyl pseudodifferential operators in terms of the Grossmann-Royer transform (Definition 6 and Lemma 4), and recover the main relations between them (Lemmas 5 and 6). Thereafter we give an exposition of known results concerning the continuity of lo- 
calization operators on modulation spaces (Subsection 5.1), and their compactness properties when the weights are chosen to be polynomial or exponential (Subsection 5.2). As a rule, we only sketch the proofs, and use the Grossmann-Royer transform formalism whenever convenient.

Finally, in Section 6 we point out some directions that might be relevant for future investigations. This includes product formulas, multilinear localization operators, continuity over quasianalytic classes, and extension to quasi-Banach modulation spaces.

\subsection{Notation}

We define $x y=x \cdot y$, the scalar product on $\mathbb{R}^{d}$. Given a vector $x=\left(x_{1}, \ldots, x_{d}\right) \in$ $\mathbb{R}^{d}$, the partial derivative with respect to $x_{j}$ is denoted by $\partial_{j}=\frac{\partial}{\partial x_{j}}$. Given a multiindex $p=\left(p_{1}, \ldots, p_{d}\right) \geq 0$, i.e., $p \in \mathbb{N}_{0}^{d}$ and $p_{j} \geq 0$, we write $\partial^{p}=\partial_{1}^{p_{1}} \cdots \partial_{d}^{p_{d}}$ and $x^{p}=\left(x_{1}, \ldots, x_{d}\right)^{\left(p_{1}, \ldots, p_{d}\right)}=\prod_{i=1}^{d} x_{i}^{p_{i}}$. We write $h \cdot|x|^{1 / \alpha}=\sum_{i=1}^{d} h_{i}\left|x_{i}\right|^{1 / \alpha_{i}}$. Moreover, for $p \in \mathbb{N}_{0}^{d}$ and $\alpha \in \mathbb{R}_{+}^{d}$, we set $(p !)^{\alpha}=\left(p_{1} !\right)^{\alpha_{1}} \ldots\left(p_{d} !\right)^{\alpha_{d}}$, while as standard $p !=$ $p_{1} ! \ldots p_{d} !$. In the sequel, a real number $r \in \mathbb{R}_{+}$may play the role of the vector with constant components $r_{j}=r$, so for $\alpha \in \mathbb{R}_{+}^{d}$, by writing $\alpha>r$ we mean $\alpha_{j}>r$ for all $j=1, \ldots, d$.

The Fourier transform is normalized to be $\hat{f}(\omega)=\mathscr{F} f(\omega)=\int f(t) e^{-2 \pi i t \omega} d t$. We use the brackets $\langle f, g\rangle$ to denote the extension of the inner product $\langle f, g\rangle=$ $\int f(t) \overline{g(t)} d t$ on $L^{2}\left(\mathbb{R}^{d}\right)$ to the dual pairing between a test function space $\mathscr{A}$ and its dual $\mathscr{A}^{\prime}:\langle\cdot, \cdot\rangle=\mathscr{A}^{\prime}\langle\cdot,,\rangle_{\mathscr{A}}$.

By $\breve{f}$ we denote the reflection $\breve{f}(x)=f(-x)$, and $\langle\cdot\rangle^{s}$ are polynomial weights

$$
\langle(x, \omega)\rangle^{s}=\langle z\rangle^{s}=\left(1+x^{2}+\omega^{2}\right)^{s / 2}, \quad z=(x, \omega) \in \mathbb{R}^{2 d}, \quad s \in \mathbb{R},
$$

and $\langle x\rangle=\left\langle 1+|x|^{2}\right\rangle^{1 / 2}$, when $x \in \mathbb{R}^{d}$.

Translation and modulation operators, $T$ and $M$ are defined by

$$
T_{x} f(\cdot)=f(\cdot-x) \quad \text { and } \quad M_{x} f(\cdot)=e^{2 \pi i x \cdot} f(\cdot), \quad x \in \mathbb{R}^{d} .
$$

The following relations hold

$$
M_{y} T_{x}=e^{2 \pi i x \cdot y} T_{x} M_{y}, \quad\left(T_{x} f\right)^{\wedge}=M_{-x} \hat{f},\left(M_{x} f\right)^{\wedge}=T_{x} \hat{f}, \quad x, y \in \mathbb{R}^{d}, f, g \in L^{2}\left(\mathbb{R}^{d}\right) .
$$

The singular values $\left\{s_{k}(L)\right\}_{k=1}^{\infty}$ of a compact operator $L \in B\left(L^{2}\left(\mathbb{R}^{d}\right)\right)$ are the eigenvalues of the positive self-adjoint operator $\sqrt{L^{*} L}$. For $1 \leq p<\infty$, the Schatten class $S_{p}$ is the space of all compact operators whose singular values lie in $l^{p}$. For consistency, we define $S_{\infty}:=B\left(L^{2}\left(\mathbb{R}^{d}\right)\right)$ to be the space of bounded operators on $L^{2}\left(\mathbb{R}^{d}\right)$. In particular, $S_{2}$ is the space of Hilbert-Schmidt operators, and $S_{1}$ is the space of trace class operators. 
Throughout the paper, we shall use the notation $A \lesssim B$ to indicate $A \leq c B$ for a suitable constant $c>0$, whereas $A \asymp B$ means that $c^{-1} A \leq B \leq c A$ for some $c \geq 1$. The symbol $B_{1} \hookrightarrow B_{2}$ denotes the continuous and dense embedding of the topological vector space $B_{1}$ into $B_{2}$.

\subsection{Basic spaces}

In general a weight $w(\cdot)$ on $\mathbb{R}^{d}$ is a non-negative and continuous function. By $L_{w}^{p}\left(\mathbb{R}^{d}\right), p \in[1, \infty]$ we denote the weighted Lebesgue space defined by the norm

$$
\|f\|_{L_{w}^{p}}=\|f w\|_{L^{p}}=\left(\int|f(x)|^{p} w(x)^{p} d x\right)^{1 / p}
$$

with the usual modification when $p=\infty$.

Similarly, the weighted mixed-norm space $L_{w}^{p, q}\left(\mathbb{R}^{2 d}\right), p, q \in[1, \infty]$ consists of (Lebesgue) measurable functions on $\mathbb{R}^{2 d}$ such that

$$
\|F\|_{L_{w}^{p, q}}=\left(\int_{\mathbb{R}^{d}}\left(\int_{\mathbb{R}^{d}}|F(x, \omega)|^{p} w(x, \omega)^{p} d x\right)^{q / p} d \omega\right)^{1 / q}<\infty .
$$

where $w(x, \omega)$ is a weight on $\mathbb{R}^{2 d}$.

In particular, when $w(x, \omega)=\langle x\rangle^{t}\langle\omega\rangle^{s}, s, t \in \mathbb{R}$, we use the notation $L_{w}^{p, q}\left(\mathbb{R}^{2 d}\right)=$ $L_{t, s}^{p, q}\left(\mathbb{R}^{2 d}\right)$, and when $w(x)=\langle x\rangle^{t}, t \in \mathbb{R}$, we use the notation $L_{t}^{p}\left(\mathbb{R}^{d}\right)$ instead.

The space of smooth functions with compact support on $\mathbb{R}^{d}$ is denoted by $\mathscr{D}\left(\mathbb{R}^{d}\right)$. The Schwartz class is denoted by $\mathscr{S}\left(\mathbb{R}^{d}\right)$, the space of tempered distributions by $\mathscr{S}^{\prime}\left(\mathbb{R}^{d}\right)$. Recall, $\mathscr{S}\left(\mathbb{R}^{d}\right)$ is a Fréchet space, the projective limit of spaces $\mathscr{S}_{p}\left(\mathbb{R}^{d}\right)$, $p \in \mathbb{N}_{0}$, defined by the norms:

$$
\|\varphi\|_{\mathscr{S}_{p}}=\sup _{|\alpha| \leq p}\left(1+|x|^{2}\right)^{p / 2}\left|\partial^{\alpha} \phi(x)\right|<\infty, \quad p \in \mathbb{N}_{0} .
$$

Note that $\mathscr{D}\left(\mathbb{R}^{d}\right) \hookrightarrow \mathscr{S}\left(\mathbb{R}^{d}\right)$.

The spaces $\mathscr{S}\left(\mathbb{R}^{d}\right)$ and $\mathscr{S}^{\prime}\left(\mathbb{R}^{d}\right)$ play an important role in various applications since the Fourier transform is a topological isomorphism between $\mathscr{S}\left(\mathbb{R}^{d}\right)$ and $\mathscr{S}\left(\mathbb{R}^{d}\right)$ which extends to a continuous linear transform from $\mathscr{S}^{\prime}\left(\mathbb{R}^{d}\right)$ onto itself.

In order to deal with particular problems in applications different generalizations of the Schwartz type spaces were proposed. An example is given by the Gevrey classes given below. Gelfand-Shilov spaces are another important example, see Section 3.

By $\Omega$ we denote an open set in $\mathbb{R}^{d}$, and $K \Subset \Omega$ means that $K$ is compact subset in $\Omega$. For $1<s<\infty$ we define the Gevrey class $G^{s}(\Omega)$ by

$$
G^{s}(\Omega)=\left\{\phi \in C^{\infty}(\Omega)\left|(\forall K \Subset \Omega)(\exists C>0)(\exists h>0) \sup _{x \in K}\right| \partial^{\alpha} \phi(x)\left|\leq C h^{|\alpha|}\right| \alpha \mid !^{s}\right\} .
$$


We denote by $G_{0}^{s}(\Omega)$ a subspace of $G^{S}(\Omega)$ which consists of compactly supported functions. We have $\mathscr{A}(\Omega) \hookrightarrow \cap_{s>1} G^{s}(\Omega)$ and $\cup_{s \geq 1} G^{S}(\Omega) \hookrightarrow C^{\infty}(\Omega)$, where $\mathscr{A}(\Omega)$ denotes the space of analytic functions defined by

$\mathscr{A}(\Omega)=\left\{\phi \in C^{\infty}(\Omega)\left|(\forall K \Subset \Omega)(\exists C>0)(\exists h>0) \quad \sup _{x \in K}\right| \partial^{\alpha} \phi(x)\left|\leq C h^{|\alpha|}\right| \alpha \mid !\right\}$.

We end this section with test function spaces for the spaces of ultradistributions due to Komatsu [46]. In fact, both the Gevrey classes and the Gelfand-Shilov spaces can be viewed as particular cases of Komatsu's construction. Let there be given an open set $\Omega \subset \mathbb{R}^{d}$, and a sequence $\left(N_{q}\right)_{q \in \mathbb{N}_{0}}$ which satisfies (M.1) and (M.2), see Section 3. The function $\phi \in C^{\infty}(\Omega)$ is called ultradifferentiable function of Beurling class $\left(N_{q}\right)$ (respectively of Roumieu class $\left\{N_{q}\right\}$ ) if, for any $K \subset \subset \Omega$ and for any $h>0$ (respectively for some $h>0$ ),

$$
\|\phi\|_{N_{q}, K, h}=\sup _{x \in K, \alpha \in \mathbb{N}_{0}^{d}} \frac{\left|\partial^{\alpha} \phi(x)\right|}{h^{|\alpha|} N_{|\alpha|}}<\infty .
$$

We say that $\phi \in \mathscr{E}^{N_{q}, K, h}(\Omega)$ if $\|\phi\|_{N_{q}, K, h}<1$ for given $K$ and $h>0$, and define the following spaces of ultradifferentiable test functions:

$$
\begin{aligned}
& \mathscr{E}^{\left(N_{q}\right)}(\Omega):=\operatorname{proj} \lim _{K \subset \subset \Omega} \operatorname{proj} \lim _{h \rightarrow 0} \mathscr{E}^{N_{q}, K, h}(\Omega) ; \\
& \mathscr{E}^{\left\{N_{q}\right\}}(\Omega):=\operatorname{proj} \lim _{K \subset \subset \Omega} \text { ind } \lim _{h \rightarrow \infty} \mathscr{E}^{N_{q}, K, h}(\Omega) .
\end{aligned}
$$

\section{The Grossmann- Royer transform}

In this section we consider the Grossmann-Royer operator $R f(x, \omega)$ and introduce the corresponding transform. We refer to [23] for the basic properties of $R f(x, \omega)$ and its relation to the Heiseberg-Weyl operator. In fact, the Grossmann-Royer operator can be viewed as a kind of reflection (therefore we use the letter $R$ to denote it), more precisely the conjugate of a reflection operator by a Heisengerg-Weyl operator, and the product formula ([23, Proposition 150]) reveals that the product of two reflections is a translation. Moreover, it satisfies the symplectic covariance property, [23, Proposition 150].

Those physically plausible interpretations motivate us to define the corresponding transform, which is just the cross-Wigner distribution in disguise. As we shall see, in many situations it is more convenient to choose the the Grossmann-Royer transform instead of some of its relatives, the cross-Wigner distribution, the crossambiguity function and the short-time Fourier transform.

The Grossmann-Royer operator $R: L^{2}\left(\mathbb{R}^{d}\right) \rightarrow L^{2}\left(\mathbb{R}^{2 d}\right)$ is given by

$$
R f(x, \omega)=R(f(t))(x, \omega)=e^{4 \pi i \omega(t-x)} f(2 x-t), \quad f \in L^{2}\left(\mathbb{R}^{d}\right), x, \omega \in \mathbb{R}^{d} .
$$


Definition 1. Let there be given $f, g \in L^{2}\left(\mathbb{R}^{d}\right)$. The Grossmann-Royer transform of $f$ and $g$ is given by

$$
R_{g} f(x, \omega)=R(f, g)(x, \omega)=\langle R f, g\rangle=\int e^{4 \pi i \omega(t-x)} f(2 x-t) \overline{g(t)} d t, \quad x, \omega \in \mathbb{R}^{d} .
$$

The short-time Fourier transform of $f$ with respect to the window $g$ is given by

$$
V_{g} f(x, \omega)=\int e^{-2 \pi i t \omega} f(t) \overline{g(t-x)} d t, \quad x, \omega \in \mathbb{R}^{d} .
$$

The cross-Wigner distribution of $f$ and $g$ is

$$
W(f, g)(x, \omega)=\int e^{-2 \pi i \omega t} f\left(x+\frac{t}{2} \overline{g\left(x-\frac{t}{2}\right)} d t, \quad x, \omega \in \mathbb{R}^{d},\right.
$$

and the cross-ambiguity function of $f$ and $g$ is

$$
A(f, g)(x, \omega)=\int e^{-2 \pi i \omega t} f\left(t+\frac{x}{2} \overline{g\left(t-\frac{x}{2}\right)} d t, \quad x, \omega \in \mathbb{R}^{d} .\right.
$$

Note that $R(f(t))(x, \omega)=e^{-4 \pi i \omega x} M_{2 \omega}\left(T_{2 x} f\right)^{\nu}(t)$ so that

$$
R_{g} f(x, \omega)=e^{-4 \pi i \omega x}\left\langle M_{2 \omega}\left(T_{2 x} f\right)^{\nu}, \bar{g}\right\rangle .
$$

Lemma 1. Let $f, g \in L^{2}\left(\mathbb{R}^{d}\right)$. Then we have:

$$
\begin{aligned}
W(f, g)(x, \omega) & =2^{d} R_{g} f(x, \omega), \\
V_{g} f(x, \omega) & =e^{-\pi i x \omega} R_{\check{g}} f\left(\frac{x}{2}, \frac{\omega}{2}\right), \\
A(f, g)(x, \omega) & =R_{\breve{g}} f\left(\frac{x}{2}, \frac{\omega}{2}\right), \quad x, \omega \in \mathbb{R}^{d} .
\end{aligned}
$$

Proof. $W(f, g)(x, \omega)=2^{d} R_{g} f(x, \omega)$ immediately follows from the change of variables $t \mapsto 2(x-t)$ in (6), so the cross-Wigner distribution and the Grossmann-Royer transform are essentially the same.

The change of variables $x-t \mapsto s$ in (5) gives

$$
\begin{aligned}
V_{g} f(x, \omega) & =\int e^{-2 \pi i(x-s) \omega} f(x-s) \overline{\check{g}(s)} d s \\
& =\int e^{4 \pi i \frac{\omega}{2}(s-x)} f\left(2 \frac{x}{2}-s\right) \overline{\check{g}(s)} d s \\
& =e^{-\pi i x \omega} \int e^{4 \pi i \frac{\omega}{2}\left(s-\frac{x}{2}\right)} f\left(2 \frac{x}{2}-s\right) \overline{\check{g}(s)} d s \\
& =e^{-\pi i x \omega} R_{\breve{g}} f\left(\frac{x}{2}, \frac{\omega}{2}\right) .
\end{aligned}
$$

Therefore, $R_{g} f(x, \omega)=e^{\pi i x \omega} V_{\breve{g}} f(2 x, 2 \omega)$. Finally, the change of variables $t-x / 2 \mapsto$ $-s$ in (7) gives 
The Grossmann-Royer transform,...

$$
\begin{aligned}
A(f, g)(x, \omega) & =\int e^{2 \pi i \omega\left(s-\frac{x}{2}\right)} f(x-s) \overline{\check{g}(s)} d s \\
& =\int e^{4 \pi i \frac{\omega}{2}\left(s-\frac{x}{2}\right)} f\left(2 \frac{x}{2}-s\right) \overline{\check{g}(s)} d s \\
& =R_{\breve{g}} f\left(\frac{x}{2}, \frac{\omega}{2}\right) .
\end{aligned}
$$

Therefore $R_{g} f(x, \omega)=A(f, \check{g})(2 x, 2 \omega)$.

By Lemma 1 we recapture the well-known formulas ([38, 88]:

$$
\begin{gathered}
A(f, g)(x, \omega)=e^{\pi i x \omega} V_{g} f(x, \omega), \\
W(f, g)(x, \omega)=2^{d} e^{4 \pi i x \omega} V_{\breve{g}} f(2 x, 2 \omega), \\
W(f, g)(x, \omega)=(\mathscr{F} A(f, g))(x, \omega), \quad x, \omega \in \mathbb{R}^{d} .
\end{gathered}
$$

The quadratic expressions $A f:=A(f, f)$ and $W f:=W(f, f)$ are called the (radar) ambiguity function and the Wigner distribution of $f$.

We collect the elementary properties of the Grossmann-Royer transform in the next proposition.

Proposition 1. Let $f, g \in L^{2}\left(\mathbb{R}^{d}\right)$. The Grossmann-Royer operator is self-adjoint, uniformly continuous on $\mathbb{R}^{2 d}$, and the following properties hold:

1. $\left\|R_{g} f\right\|_{\infty} \leq\|f\|\|g\|$;

2. $R_{g} f=\overline{R_{f} g}$;

3. The covariance property:

$$
R_{T_{x} M_{\omega} g} T_{x} M_{\omega} f(p, q)=R_{g} f(p-x, q-\omega), \quad x, \omega, p, q \in \mathbb{R}^{d} ;
$$

4. $R_{\hat{g}} \hat{f}(x, \omega)=R_{g} f(-\omega, x)$;

5. For $f_{1}, f_{2}, g_{1}, g_{2} \in L^{2}\left(\mathbb{R}^{d}\right)$, the Moyal identity holds:

$$
\left\langle R_{g_{1}} f_{1}, R_{g_{2}} f_{2}\right\rangle=\left\langle f_{1}, f_{2}\right\rangle \overline{\left\langle g_{1}, g_{2}\right\rangle} .
$$

6. $R_{g} f$ maps $\mathscr{S}\left(\mathbb{R}^{d}\right) \times \mathscr{S}\left(\mathbb{R}^{d}\right)$ into $\mathscr{S}\left(\mathbb{R}^{d} \times \mathbb{R}^{d}\right)$ and extends to a map from $\mathscr{S}^{\prime}\left(\mathbb{R}^{d}\right) \times \mathscr{S}^{\prime}\left(\mathbb{R}^{d}\right)$ into $\mathscr{S}^{\prime}\left(\mathbb{R}^{d} \times \mathbb{R}^{d}\right)$.

Proof. We first show that $\langle R f, g\rangle=\langle f, R g\rangle$ :

$$
\begin{aligned}
\langle R f, g\rangle & =\int e^{4 \pi i \omega(t-x)} f(2 x-t) \overline{g(t)} d t=\int e^{4 \pi i \omega(x-s)} f(s) \overline{g(2 x-s)} d s \\
& =\int f(s) \overline{e^{4 \pi i \omega(s-x)} g(2 x-s)} d s=\langle f, R g\rangle .
\end{aligned}
$$

The uniform continuity and the estimate in 1 . follow from (8), the continuity of translation, modulation and reflection operators on $L^{2}\left(\mathbb{R}^{d}\right)$ and the CauchySchwartz inequality.

The property 2 . follows from the self-adjointness. 
For the covariance we have:

$$
\begin{aligned}
R_{T_{x} M_{\omega} g} T_{x} M_{\omega} f(p, q) & =\int e^{4 \pi i q(t-p)} T_{x} M_{\omega} f(2 p-t) \overline{T_{x} M_{\omega} g}(t) d t \\
& =\int e^{4 \pi i q(t-p)} e^{2 \pi i \omega(2 p-t)} f(2 p-t-x) e^{-2 \pi i \omega t} \bar{g}(t-x) d t \\
& =\int e^{4 \pi i q(s+x-p)} e^{2 \pi i \omega(2 p-s-x)} f(2(p-x)-s) e^{-2 \pi i \omega(s+x)} \bar{g}(s) d s \\
& =\int e^{4 \pi i(q-\omega)(s-(p-x))} f(2(p-x)-s) \bar{g}(s) d s \\
& =R_{g} f(p-x, q-\omega), \quad x, \omega, p, q \in \mathbb{R}^{d} .
\end{aligned}
$$

To prove 4 . we note that the integrals below are absolutely convergent and that the change of order of integration is allowed. Moreover, when suitably interpreted, certain oscillatory integrals are meaningful in $\mathscr{S}^{\prime}\left(\mathbb{R}^{d}\right)$. We refer to e.g. $[65,88]$ for such interpretation. In particular, if $\delta$ denotes the Dirac distribution, then the Fourier inversion formula in the sense of distributions gives $\int e^{2 \pi i x \omega} d \omega=\delta(x)$, wherefrom $\iint \phi(x) e^{2 \pi i(x-y) \omega} d x d \omega=\phi(y)$, when $\phi \in \mathscr{S}\left(\mathbb{R}^{d}\right)$.

Therefore we have:

$$
\begin{aligned}
R_{\hat{g}} \hat{f}(x, \omega) & =\int e^{4 \pi i \omega(t-x)} \hat{f}(2 x-t) \overline{\hat{g}(t)} d t \\
& =e^{-4 \pi i \omega x} \iiint e^{4 \pi i \omega t} e^{-2 \pi i \eta(2 x-t)} f(\eta) e^{2 \pi i y t} \overline{g(y)} d \eta d y d t \\
& =e^{-4 \pi i \omega x} \iiint e^{-2 \pi i t(-2 \omega-\eta-y)} e^{-4 \pi i \eta x} f(\eta) \overline{g(y)} d \eta d y d t \\
& =e^{-4 \pi i \omega x} \iint \delta(-2 \omega-\eta-y) e^{-4 \pi i \eta x} f(\eta) \overline{g(y)} d \eta d y \\
& =e^{-4 \pi i \omega x} \int e^{-4 \pi i(-2 \omega-y) x} f(-2 \omega-y) \overline{g(y)} d y \\
& =\int e^{4 \pi i(y+\omega) x} f(-2 \omega-y) \overline{g(y)} d y \\
& =R_{g} f(-\omega, x), \quad x, \omega \in \mathbb{R}^{d} .
\end{aligned}
$$

For the Moyal identity, we again use the Fourier transform of Dirac's delta:

$$
\begin{aligned}
\left\langle R_{g_{1}} f_{1}, R_{g_{2}} f_{2}\right\rangle & =\int_{\mathbb{R}^{2 d}}\left(\int e^{4 \pi i \omega(t-x)} f_{1}(2 x-t) \overline{g_{1}(t)} d t\right. \\
& \times \int e^{\left.-4 \pi i \omega(s-x) \overline{f_{2}}(2 x-s) g_{2}(s) d s\right) d x d \omega} \\
& =\int_{\mathbb{R}^{2 d}} \iint e^{-2 \pi i \omega(-2(t-s))} f_{1}(2 x-t) \overline{f_{2}}(2 x-s) \overline{g_{1}(t)} g_{2}(s) d t d s d x d \omega \\
& =\iiint \delta(-2(t-s)) f_{1}(2 x-t) \overline{f_{2}}(2 x-s) \overline{g_{1}(t)} g_{2}(s) d t d s d x \\
& =\left\langle f_{1}, f_{2}\right\rangle \overline{\left\langle g_{1}, g_{2}\right\rangle} .
\end{aligned}
$$


To prove 6. we note that the Grossman-Royer transform can be written in the form

$$
R_{g} f(x, \omega)=\left(\mathscr{F}_{2} \circ \tau^{*}\right) f \otimes \bar{g}(x, \omega), \quad x, \omega \in \mathbb{R}^{d},
$$

where $\mathscr{F}_{2}$ denotes the partial Fourier transform with respect to the second variable, and $\tau^{*}$ is the pullback of the operator $\tau:(x, s) \mapsto(2 x-s, s)$. The theorem now follows from the invariance of $\mathscr{S}\left(\mathbb{R}^{2 d}\right)$ under $\tau^{*}$ and $\mathscr{F}_{2}$, and $f \otimes \bar{g} \in \mathscr{S}\left(\mathbb{R}^{2 d}\right)$ when $f, g \in \mathscr{S}\left(\mathbb{R}^{d}\right)$, and the extension to tempered distributions is straightforward.

The Moyal identity formula implies the inversion formula:

$$
f=\frac{1}{\left\langle g_{2}, g_{1}\right\rangle} \iint R_{g_{1}} f(x, \omega) \operatorname{Rg}_{2}(x, \omega) d x d \omega,
$$

We refer to [38] for details (explained in terms of the short-time Fourier transform).

For the Fourier transform of $R_{g} f$ we have:

Proposition 2. Let $f, g \in L^{2}\left(\mathbb{R}^{d}\right)$. Then $\mathscr{F}\left(R_{g} f\right)(x, \omega)=R_{\check{g}} f\left(-\frac{\omega}{2}, \frac{x}{2}\right), x, \omega \in \mathbb{R}^{d}$.

Proof. We again use the interpretation of oscillatory integrals in distributional sense.

$$
\begin{aligned}
\mathscr{F}\left(R_{g} f\right)(x, \omega) & =\iint e^{-2 \pi i\left(x x^{\prime}+\omega \omega^{\prime}\right)} R_{g} f\left(x^{\prime}, \omega^{\prime}\right) d x^{\prime} d \omega^{\prime} \\
& =\iiint e^{-2 \pi i\left(x x^{\prime}+\omega \omega^{\prime}\right)+4 \pi i \omega^{\prime}\left(t-x^{\prime}\right)} f\left(2 x^{\prime}-t\right) \bar{g}(t) d t d x^{\prime} d \omega^{\prime} \\
& =\iiint e^{-2 \pi i \omega^{\prime}\left(\omega-2 t+2 x^{\prime}\right)} e^{-2 \pi i x x^{\prime}} f\left(2 x^{\prime}-t\right) \bar{g}(t) d t d x^{\prime} d \omega^{\prime} \\
& =\iint \delta\left(\omega-2 t+2 x^{\prime}\right) e^{-2 \pi i x x^{\prime}} f\left(2 x^{\prime}-t\right) \bar{g}(t) d t d x^{\prime} \\
& =\iint e^{-2 \pi i x(t-\omega / 2)} f(t-\omega) \bar{g}(t) d t \\
& =\iint e^{-4 \pi i \frac{x}{2}(t-\omega / 2)} f(t-\omega) \bar{g}(t) d t \\
& =\iint e^{4 \pi i \frac{x}{2}(s-(-\omega / 2))} f(2 \cdot(-\omega / 2)-s) \bar{g}(s) d s \\
& =R_{\check{g}} f\left(-\frac{\omega}{2}, \frac{x}{2}\right), \quad x, \omega \in \mathbb{R}^{d},
\end{aligned}
$$

which proves the proposition.

The following lemma shows that the Grossmann-Royer transform yields the correct marginal densities. This follows from the well-known marginal densities of the (cross-)Wigner distribution, since the transforms are essentially the same. However, we give an independent proof below. We refer to $[38,23]$ for the discussion on the probabilistic interpretation of the (cross-)Wigner distribution.

Lemma 2. Let $f, g \in L^{1} \mathbb{R}^{d} \cap L^{2} \mathbb{R}^{d}$. Then 


$$
\begin{gathered}
\int_{\mathbb{R}^{d}} R_{g} f(x, \omega) d \omega=2^{-d} f(x) \bar{g}(x), \\
\int_{\mathbb{R}^{d}} R_{g} f(x, \omega) d x=2^{-d} \hat{f}(\omega) \overline{\hat{g}}(\omega),
\end{gathered}
$$

and, in particular $2^{d} \int R_{f} f(x, \omega) d \omega=|f(x)|^{2}$, and $2^{d} \int R_{f} f(x, \omega) d x=|\hat{f}(\omega)|^{2}$.

Proof. We will use the change of variables $s=x-t$ to obtain

$$
\begin{aligned}
\int_{\mathbb{R}^{d}} R_{g} f(x, \omega) d \omega & =\iint e^{4 \pi i \omega(t-x)} f(2 x-t) \bar{g}(t) d t d \omega \\
& =\iint e^{-2 \pi i \omega(2 s)} f(x+s) \bar{g}(x-s) d s d \omega \\
& =\int \delta(2 s) f(x+s) \bar{g}(x-s) d s \\
& =2^{-d} f(x) \bar{g}(x), \quad x \in \mathbb{R}^{d}
\end{aligned}
$$

and similarly

$$
\begin{aligned}
\int_{\mathbb{R}^{d}} R_{g} f(x, \omega) d x & =\iint e^{-2 \pi i \omega(2 x-2 t)} f(2 x-t) \bar{g}(t) d t d x \\
& =\iint e^{-2 \pi i \omega(2 x-t)} f(2 x-t) \overline{e^{-2 \pi i \omega t} g(t)} d t d x \\
& =2^{-d} \hat{f}(\omega) \overline{\hat{g}}(\omega), \quad \omega \in \mathbb{R}^{d}
\end{aligned}
$$

The particular case is obvious.

Remark 1. If $f$ belongs to the dense subspace of $L^{2}\left(\mathbb{R}^{d}\right)$ such that $R_{f} f \in L^{1}\left(\mathbb{R}^{2 d}\right)$ and the Fubini theorem holds, then Plancherel's theorem follows from Lemma 2:

$$
\|\hat{f}\|^{2}=2^{d} \iint R_{f} f(x, \omega) d x d \omega=2^{d} \iint R_{f} f(x, \omega) d \omega d x=\|f\|^{2}
$$

We end the section with the weak form of the uncertainty principle for the Grossmann-Royer transform.

Proposition 3. Let there be given $f, g \in L^{2}\left(\mathbb{R}^{d}\right) \backslash 0$, and let $U \subset \mathbb{R}^{2 d}$ and $\varepsilon>0$ be such that

$$
\int_{U}\left|R_{g} f(x, \omega)\right|^{2} d x d \omega \geq(1-\varepsilon)\|f\|\|g\| \text {. }
$$

Then $|U| \geq 1-\varepsilon$.

Proof. From Proposition 1 1. we have

$$
(1-\varepsilon)\|f\|\|g\| \leq \int_{U}\left|R_{g} f(x, \omega)\right|^{2} d x d \omega \leq\left\|R_{g} f\right\|_{\infty}|U| \leq\|f\|\|g\||U|,
$$

and the claim follows. 
We end this section with the relation between the Grossmann-Royer operator and the Heisenberg-Weyl operator, also known as displacement operators since it describes translations in phase space. Notice that in [25] the Grossmann-Royer operators are defined in terms of the Heisenberg-Weyl operators, so our definition (3) is formulated as a proposition there.

Definition 2. Let there be given $f, g \in L^{2}\left(\mathbb{R}^{d}\right)$. The Heisenberg-Weyl operator $T$ : $L^{2}\left(\mathbb{R}^{d}\right) \rightarrow L^{2}\left(\mathbb{R}^{2 d}\right)$ is given by

$$
T f(x, \omega)=T(f(t))(x, \omega)=e^{2 \pi i \omega\left(t-\frac{x}{2}\right)} f(t-x), \quad x, \omega \in \mathbb{R}^{d},
$$

and the Heisenberg-Weyl transform is defined to be

$$
T_{g} f(x, \omega)=T(f, g)(x, \omega)=\langle T f, g\rangle=\int e^{2 \pi i \omega\left(t-\frac{x}{2}\right)} f(t-x) \overline{g(t)} d t, \quad x, \omega \in \mathbb{R}^{d} .
$$

Proposition 4. Let there be given $f, g \in L^{2}\left(\mathbb{R}^{d}\right)$. Then we have

1. $R f(x, \omega)=T(x, \omega) R(0) T f(-x,-\omega)$;

2. $R(x, \omega) R f(p, q)=e^{-4 \pi i \sigma((x, \omega),(p, q))} T f(2(x-p, \omega-q))$, where $\sigma$ is the standard symplectic form on phase space $\mathbb{R}^{2 d}$ :

$$
\sigma((x, \omega),(p, q))=\omega \cdot p-x \cdot q .
$$

Proof. 1. Note that $R f(0)=f(-t)$, wherefrom

$$
T f(-x,-\omega)=e^{-2 \pi i \omega\left(t+\frac{x}{2}\right)} f(t+x) \Rightarrow R(0) T f(-x,-\omega)=e^{2 \pi i \omega\left(t-\frac{x}{2}\right)} f(x-t) .
$$

Hence,

$$
\begin{aligned}
T(x, \omega) R(0) T f(-x,-\omega) & =T(x, \omega) e^{2 \pi i \omega\left(t-\frac{x}{2}\right)} f(x-t) \\
& =e^{-\pi i \omega x} T\left(e^{2 \pi i \omega t} f(x-t)\right)(x, \omega) \\
& =e^{-\pi i \omega x} e^{2 \pi i \omega\left(t-\frac{x}{2}\right)} e^{2 \pi i \omega(t-x)} f(x-(t-x)) \\
& =e^{4 \pi i \omega(t-x)} f(2 x-t) \\
& =R f(x, \omega) .
\end{aligned}
$$

To prove 2. we calculate both sides:

$$
\begin{aligned}
R(x, \omega) R f(p, q) & =R\left(e^{4 \pi i q(t-p)} f(2 p-t)\right)(x, \omega) \\
& =e^{-4 \pi i q p} R\left(e^{4 \pi i q t} f(2 p-t)\right)(x, \omega) \\
& =e^{-4 \pi i q p} e^{4 \pi i \omega(t-x)} e^{4 \pi i q(2 x-t)} f(2 p-(2 x-t)) \\
& =e^{4 \pi i \omega(t-x)} e^{4 \pi i q(2 x-t-p)} f(t-2(x-p)),
\end{aligned}
$$

and 


$$
\begin{gathered}
e^{-4 \pi i(\omega p-x q)} T f(2((x, \omega)-(p, q)))=e^{-4 \pi i(\omega p-x q)} e^{2 \pi i 2(\omega-q)(t-(x-p))} f(t-2(x-p)) \\
=e^{-4 \pi i(\omega p-x q)} e^{4 \pi i(\omega-q) t} e^{-4 \pi i(\omega-q)(x-p)} f(t-2(x-p)) \\
=e^{4 \pi i \omega(-p+t-x-p)} e^{-4 \pi i p q} e^{4 \pi i q(x-t+x)} f(t-2(x-p)) \\
=e^{4 \pi i \omega(t-x)} e^{4 \pi i q(2 x-t-p)} f(t-2(x-p)),
\end{gathered}
$$

which shows that $R(x, \omega) R f(p, q)=e^{-4 \pi i \sigma((x, \omega),(p, q))} T f(2(x-p, \omega-q))$.

Finally, if $\mathscr{F}_{\sigma}$ denotes the symplectic Fourier transform in $L^{2}\left(\mathbb{R}^{2 d}\right)$ :

$$
\begin{aligned}
\mathscr{F}_{\sigma}(F(x, \omega))(p, q) & =\int_{\mathbb{R}^{2 d}} e^{-2 \pi i \sigma((x, \omega),(p, q))} F(x, \omega) d x d \omega \\
& =\int_{\mathbb{R}^{2 d}} e^{-2 \pi i(\omega p-x q)} F(x, \omega) d x d \omega
\end{aligned}
$$

then the Grossmann-Royer operator and the Heisenberg-Weyl operator are the symplectic Fourier transforms of each other.

Proposition 5. Let there be given $f, g \in L^{2}\left(\mathbb{R}^{d}\right)$ and let $\mathscr{F}_{\sigma}$ be the symplectic Fourier transform. Then

$$
R f(p, q)=2^{-d} \mathscr{F}_{\sigma}(T(f(t))(x, \omega))(-p,-q)
$$

Proof.

$$
\begin{aligned}
\mathscr{F}_{\sigma}(T(f(t))(x, \omega))(-p,-q) & =\int_{\mathbb{R}^{2 d}} e^{2 \pi i(x q-\omega p)} e^{2 \pi i \omega\left(t-\frac{x}{2}\right)} f(t-x) d x d \omega \\
& =\int_{\mathbb{R}^{d}}\left(\int_{\mathbb{R}^{d}} e^{2 \pi i \omega\left(t-p-\frac{x}{2}\right)} d \omega\right) e^{2 \pi i q x)} f(t-x) d x \\
& =\int_{\mathbb{R}^{d}} \delta\left(t-p-\frac{x}{2}\right) e^{2 \pi i q x} f(t-x) d x \\
& =2^{d} e^{4 \pi i q(t-p)} f(t-2(t-p)) d x=2^{d} R f(p, q)
\end{aligned}
$$

where we again used the Fourier inversion formula in the sence of distributions.

Since it is easy to see that $\mathscr{F}_{\sigma}$ is an involution, we also have

$$
T f(p, q)=2^{d} \mathscr{F}_{\sigma}(R(f(t))(x, \omega))(-p,-q) .
$$

Remark 2. The Weyl operator $L_{a}$ with the symbol $a$ is introduced in [25, Definition $37]$ by the means of the symplectic Fourier transform $\mathscr{F}_{\sigma} a$ of the symbol and the Heisenberg-Weyl operator:

$$
L_{a} f(t)=\int_{\mathbb{R}^{2 d}} \mathscr{F}_{\sigma} a(x, \omega) T(f(t))(x, \omega) d x d \omega .
$$

There it is shown that such definition coincides with the usual one, see Section 5, as well as with the representation given by Lemma 5 . 


\section{Gelfand-Shilov spaces}

Problems of regularity of solutions to partial differential equations (PDEs) play a central role in the modern theory of PDEs. When solutions of certain PDEs are smooth but not analytic, several intermediate spaces of functions are proposed in order to describe its decrease at infinity and also the regularity in $\mathbb{R}^{d}$. In particular, in the study of properties of solutions of certain parabolic initial-value problems Gelfand and Shilov introduced the spaces of type $S$ in [35]. Such spaces provide uniqueness and correctness classes for solutions of Cauchy problems, [36]. We refer to [36] for the fundamental properties of such spaces which are afterwards called Gelfand-Shilov spaces.

More recently, Gelfand-Shilov spaces were used in $[9,10]$ to describe exponential decay and holomorphic extension of solutions to globally elliptic equations, and in [48] in the regularizing properties of the Boltzmann equation. We refer to [52] for a recent overview and for applications in quantum mechanics and traveling waves, and to [78] for the properties of the Bargmann transform on Gelfand-Shilov spaces. The original definition is generalized already in [36, Ch. IV, App. 1], and more general decay and regularity conditions can be systematically studied by using Komatsu's approach to ultradifferentiable functions developed in [46]. An interesting extension based on the iterates of the harmonic oscillator is studied in [80] under the name Pilipović spaces, see also [54].

In the context of time-frequency analysis, certian Gelfand-Shilov spaces can be described in terms of modulation spaces $[38,43]$. The corresponding pseudodifferential calculus is developed in [78, 79]. Gelfand-Shilov spaces are also used in the study of time-frequency localization operators in $[18,19,70]$, thus extending the context of the pioneering results of Cordero and Grochenig [13].

In this section we introduce Gelfand-Shilov spaces and list important equivalent characterizations. We also present the kernel theorem which will be used in the study of localization operators.

\subsection{Definition}

The regularity and decay properties of elements of Gelfand-Shilov spaces are initially measured with respect to sequences of the form $M_{p}=p^{\alpha p}, p \in \mathbb{N}, \alpha>0$ or, equivalently, the Gevrey sequences $M_{p}=p !^{\alpha}, p \in \mathbb{N}, \alpha>0$.

We follow here Komatsu's approach [46] to spaces of ultra-differentiable functions to extend the original definition as follows.

Let $\left(M_{p}\right)_{p \in \mathbb{N}_{0}}$ be a sequence of positive numbers monotonically increasing to infinity which satisfies:

$(M .1) \quad M_{p}^{2} \leq M_{p-1} M_{p+1}, \quad p \in \mathbb{N}$

(M.2) There exist positive constants $A, H$ such that

$$
M_{p} \leq A H^{p} \min _{0 \leq q \leq p} M_{p-q} M_{q}, p, q \in \mathbb{N}_{0},
$$


or, equivalently, there exist positive constants $A, H$ such that

$$
M_{p+q} \leq A H^{p+q} M_{p} M_{q}, p, q \in \mathbb{N}_{0} ;
$$

We assume $M_{0}=1$, and that $M_{p}^{1 / p}$ is bounded below by a positive constant.

Remark 3. To give an example, we describe (M.1) and (M.2) as follows. Let $\left(s_{p}\right)_{p \in \mathbb{N}_{0}}$ be a sequence of positive numbers monotonically increasing to infinity $\left(s_{p} \nearrow_{\infty}\right)$ so that for every $p, q \in \mathbb{N}_{0}$ there exist $A, H>0$ such that

$$
\prod_{j=1}^{q} s_{p+j}=s_{p+1} \cdots s_{p+q} \leq A H^{p} s_{1} \cdots s_{q}=A H^{p} \prod_{j=1}^{q} s_{j} .
$$

Then the sequence $\left(S_{p}\right)_{p \in \mathbb{N}_{0}}$ given by $S_{p}=\prod_{j=1}^{p} s_{j}, S_{0}=1$, satisfies $(M .1)$ and (M.2).

Conversely, if $\left(S_{p}\right)_{p \in \mathbb{N}_{0}}$ given by $S_{p}=\prod_{j=1}^{p} s_{j}, s_{j}>0, j \in \mathbb{N}, S_{0}=1$, satisfies (M.1) then $\left(s_{p}\right)_{p \in \mathbb{N}_{0}}$ increases to infinity, and if it satisfies (M.2) then (12) holds.

Let $\left(M_{p}\right)_{p \in \mathbb{N}_{0}}$ and $\left(N_{q}\right)_{q \in \mathbb{N}_{0}}$ be sequences which satisfy $(M .1)$. We write $M_{p} \subset N_{q}$ $\left(\left(M_{p}\right) \prec\left(N_{q}\right)\right.$, respectively) if there are constants $H, C>0$ (for any $H>0$ there is a constant $C>0$, respectively) such that $M_{p} \leq C H^{p} N_{p}, p \in \mathbb{N}_{0}$. Also, $\left(M_{p}\right)_{p \in \mathbb{N}_{0}}$ and $\left(N_{q}\right)_{q \in \mathbb{N}_{0}}$ are said to be equivalent if $M_{p} \subset N_{q}$ and $N_{q} \subset M_{p}$ hold.

Definition 3. Let there be given sequences of positive numbers $\left(M_{p}\right)_{p \in \mathbb{N}_{0}}$ and $\left(N_{q}\right)_{q \in \mathbb{N}_{0}}$ which satisfy (M.1) and (M.2). Let $\mathscr{S}_{M_{p}, A}^{N_{q}, B}\left(\mathbb{R}^{d}\right)$ be defined by

$$
\mathscr{S}_{M_{p}, A}^{N_{q}, B}\left(\mathbb{R}^{d}\right)=\left\{f \in C^{\infty}\left(\mathbb{R}^{d}\right) \mid\left\|x^{\alpha} \partial^{\beta} f\right\|_{L^{\infty}} \leq C A^{\alpha} M_{|\alpha|} B^{\beta} N_{|\beta|}, \quad \forall \alpha, \beta \in \mathbb{N}_{0}^{d}\right\},
$$

for some positive constant $C$, where $A=\left(A_{1}, \ldots, A_{d}\right), B=\left(B_{1}, \ldots, B_{d}\right), A, B>0$.

Gelfand-Shilov spaces $\Sigma_{M_{p}}^{N_{q}}\left(\mathbb{R}^{d}\right)$ and $\mathscr{S}_{M_{p}}^{N_{q}}\left(\mathbb{R}^{d}\right)$ are projective and inductive limits of the (Fréchet) spaces $\mathscr{S}_{M_{p}, A}^{N_{q}, B}\left(\mathbb{R}^{d}\right)$ with respect to $A$ and $B$ :

$$
\Sigma_{M_{p}}^{N_{q}}\left(\mathbb{R}^{d}\right):=\operatorname{proj} \lim _{A>0, B>0} \mathscr{S}_{M_{p}, A}^{N_{q}, B}\left(\mathbb{R}^{d}\right) ; \quad \mathscr{S}_{M_{p}}^{N_{q}}\left(\mathbb{R}^{d}\right):=\text { ind } \lim _{A>0, B>0} \mathscr{S}_{M_{p}, A}^{N_{q}, B}\left(\mathbb{R}^{d}\right) .
$$

The corresponding dual spaces of $\Sigma_{M_{p}}^{N_{q}}\left(\mathbb{R}^{d}\right)$ and $\mathscr{S}_{M_{p}}^{N_{q}}\left(\mathbb{R}^{d}\right)$ are the spaces of ultradistributions of Beurling and Roumier type respectively:

$$
\begin{aligned}
& \left(\Sigma_{M_{p}}^{N_{q}}\right)^{\prime}\left(\mathbb{R}^{d}\right):=\text { ind } \lim _{A>0, B>0}\left(\mathscr{S}_{M_{p}, A}^{N_{q}, B}\right)^{\prime}\left(\mathbb{R}^{d}\right) ; \\
& \left(\mathscr{S}_{M_{p}}^{N_{q}}\right)^{\prime}\left(\mathbb{R}^{d}\right):=\operatorname{proj} \lim _{A>0, B>0}\left(\mathscr{S}_{M_{p}, A}^{N_{q}, B}\right)^{\prime}\left(\mathbb{R}^{d}\right) .
\end{aligned}
$$

Of course, for certain choices of the sequences $\left(M_{p}\right)_{p \in \mathbb{N}_{0}}$ and $\left(N_{q}\right)_{q \in \mathbb{N}_{0}}$ the spaces $\Sigma_{M_{p}}^{N_{q}}\left(\mathbb{R}^{d}\right)$ and $\mathscr{S}_{M_{p}}^{N_{q}}\left(\mathbb{R}^{d}\right)$ are trivial, i.e. they contain only the function $\phi \equiv 0$. Nontrivial Gelfand-Shilov spaces are closed under translation, dilation, multiplication with 
$x \in \mathbb{R}^{d}$, and differentiation. Moreover, they are closed under the action of certain differential operators of infinite order (ultradifferentiable operators in the terminology of Komatsu). We refer to [46] for topological properties in a more general context of test function spaces for ultradistributions.

When $\left(M_{p}\right)_{p \in \mathbb{N}_{0}}$ and $\left(N_{q}\right)_{q \in \mathbb{N}_{0}}$ are Gevrey sequences: $M_{p}=p !^{r}, p \in \mathbb{N}_{0}$ and $N_{q}=q !^{s}, q \in \mathbb{N}_{0}$, for some $r, s \geq 0$, then we use the notation

$$
\mathscr{S}_{M_{p}}^{N_{q}}\left(\mathbb{R}^{d}\right)=\mathscr{S}_{r}^{s}\left(\mathbb{R}^{d}\right) \text { and } \Sigma_{M_{p}}^{N_{q}}\left(\mathbb{R}^{d}\right)=\Sigma_{r}^{s}\left(\mathbb{R}^{d}\right) .
$$

If, in addition, $s=r$, then we put

$$
\mathscr{S}^{\{s\}}\left(\mathbb{R}^{d}\right)=\mathscr{S}_{s}^{s}\left(\mathbb{R}^{d}\right) \text { and } \Sigma^{(s)}\left(\mathbb{R}^{d}\right)=\Sigma_{s}^{s}\left(\mathbb{R}^{d}\right) .
$$

The choice of Gevrey sequences (which is the most often used choice in the literature) may serve well as an illuminating example in different contexts. In particular, when discussing the nontriviality we have the following:

1. the space $\mathscr{S}_{r}^{s}\left(\mathbb{R}^{d}\right)$ is nontrivial if and only if $s+r>1$, or $s+r=1$ and $s r>0$,

2. if $s+r \geq 1$ and $s<1$, then every $f \in \mathscr{S}_{r}^{s}\left(\mathbb{R}^{d}\right)$ can be extended to the complex domain as an entire function,

3. if $s+r \geq 1$ and $s=1$, then every $f \in \mathscr{S}_{r}^{s}\left(\mathbb{R}^{d}\right)$ can be extended to the complex domain as a holomorphic function in a strip.

4. the space $\Sigma_{r}^{s}\left(\mathbb{R}^{d}\right)$ is nontrivial if and only if $s+r>1$, or, if $s+r=1$ and $s r>0$ and $(s, r) \neq(1 / 2,1 / 2)$.

We refer to [36] or [52] for the proof in the case of $\mathscr{S}_{r}^{s}\left(\mathbb{R}^{d}\right)$, and to [54] for the spaces $\Sigma_{r}^{s}\left(\mathbb{R}^{d}\right)$, see also [78].

Whenever nontrivial, Gelfand-Shilov spaces contain "enough functions" in the following sense. A test function space $\Phi$ is "rich enough" if

$$
\int f(x) \varphi(x) d x=0, \quad \forall \varphi \in \Phi \Rightarrow f(x) \equiv 0(\text { a.e. }) .
$$

The discussion here above shows that Gelfand-Shilov classes $\mathscr{S}_{r}^{s}\left(\mathbb{R}^{d}\right)$ consist of quasi-analytic functions when $s \in(0,1)$. This is in sharp contrast with e.g. Gevrey classes $G^{s}\left(\mathbb{R}^{d}\right), s>1$, another family of functions commonly used in regularity theory of partial differential equations, whose elements are always non-quasianalytic. We refer to [61] for microlocal analysis in Gervey classes and note that $G_{0}^{s}\left(\mathbb{R}^{d}\right) \hookrightarrow \mathscr{S}_{s}^{s}\left(\mathbb{R}^{d}\right) \hookrightarrow G^{s}\left(\mathbb{R}^{d}\right), s>1$.

When the spaces are nontrivial we have dense and continuous inclusions:

$$
\Sigma_{r}^{s}\left(\mathbb{R}^{d}\right) \hookrightarrow \mathscr{S}_{r}^{s}\left(\mathbb{R}^{d}\right) \hookrightarrow \mathscr{S}\left(\mathbb{R}^{d}\right) .
$$

In fact, $\mathscr{S}\left(\mathbb{R}^{d}\right)$ can be revealed as a limiting case of $S_{r}^{S}\left(\mathbb{R}^{d}\right)$, i.e.

$$
\mathscr{S}\left(\mathbb{R}^{d}\right)=\mathscr{S}_{\infty}^{\infty}\left(\mathbb{R}^{d}\right)=\lim _{s, r \rightarrow \infty} \mathscr{S}_{r}^{s}\left(\mathbb{R}^{d}\right),
$$

when the passage to the limit $s, r \rightarrow \infty$ is interpreted correctly, see [36], page 169. 
We refer to [80] where it is shown how to overcome the minimality condition $\left(\Sigma_{1 / 2}^{1 / 2}\left(\mathbb{R}^{d}\right)=0\right)$ by transferring the estimates for $\left\|x^{\alpha} \partial^{\beta} f\right\|_{L^{\infty}}$ into the estimates of the form $\left\|H^{N} f\right\|_{L^{\infty}} \lesssim h^{N}(N !)^{2 s}$, for some (for every ) $h>0$, where $H=|x|^{2}-\Delta$ is the harmonic oscillator.

In what follows, the special role will be played by the Gelfand-Shilov space of analytic functions $\mathscr{S}^{(1)}\left(\mathbb{R}^{d}\right):=\Sigma_{1}^{1}\left(\mathbb{R}^{d}\right)$. According to Theorem 1 here below, we have

$$
f \in \mathscr{S}^{(1)}\left(\mathbb{R}^{d}\right) \Longleftrightarrow \sup _{x \in \mathbb{R}^{d}}\left|f(x) e^{h \cdot|x|}\right|<\infty \text { and } \sup _{\omega \in \mathbb{R}^{d}}\left|\hat{f}(\omega) e^{h \cdot|\omega|}\right|<\infty, \quad \forall h>0 .
$$

Any $f \in \mathscr{S}^{(1)}\left(\mathbb{R}^{d}\right)$ can be extended to a holomorphic function $f(x+i y)$ in the strip $\left\{x+i y \in \mathbb{C}^{d}:|y|<T\right\}$ some $T>0,[36,52]$. The dual space of $\mathscr{S}^{(1)}\left(\mathbb{R}^{d}\right)$ will be denoted by $\mathscr{S}^{(1)^{\prime}}\left(\mathbb{R}^{d}\right)$. In fact, $\mathscr{S}^{(1)}\left(\mathbb{R}^{d}\right)$ is isomorphic to the Sato test function space for the space of Fourier hyperfunctions $\mathscr{S}^{(1)^{\prime}}\left(\mathbb{R}^{d}\right)$, see [20].

\subsection{Equivalent conditions}

In this subsection we recall the well known equivalent characterization of GelfandShilov spaces which shows the important behavior of Gelfand-Shilov spaces under the action of the Fourier transform. Already in [36] it is shown that the Fourier transform is a topological isomorphism between $\mathscr{S}_{r}^{s}\left(\mathbb{R}^{d}\right)$ and $\mathscr{S}_{s}^{r}\left(\mathbb{R}^{d}\right)\left(\mathscr{F}_{(}\left(\mathscr{S}_{r}^{s}\right)=\mathscr{S}_{s}^{r}\right)$, which extends to a continuous linear transform from $\left(\mathscr{S}_{r}^{s}\right)^{\prime}\left(\mathbb{R}^{d}\right)$ onto $\left(\mathscr{S}_{s}^{r}\right)^{\prime}\left(\mathbb{R}^{d}\right)$. In particular, if $s=r$ and $s \geq 1 / 2$ then $\mathscr{F}\left(\mathscr{S}_{s}^{s}\right)\left(\mathbb{R}^{d}\right)=\mathscr{S}_{s}^{s}\left(\mathbb{R}^{d}\right)$. Similar assertions hold for $\Sigma_{r}^{s}\left(\mathbb{R}^{d}\right)$.

This invariance properties easily follow from the following theorem which also enlightens fundamental properties of Gelfand-Shilov spaces implicitly contained in their definition. Among other things, it states that the decay and regularity estimates of $f \in \mathscr{S}_{M_{p}}^{N_{q}}\left(\mathbb{R}^{d}\right)$ can be studied separately.

Before we state the theorem, we introduce another notion. The associated function for a given sequence $\left(M_{p}\right)$ is defined by

$$
M(\rho)=\sup _{p \in \mathbb{N}_{0}} \ln \frac{\rho^{p} M_{0}}{M_{p}}, \quad 0<\rho<\infty .
$$

For example, the associated function for the Gevrey sequence $M_{p}=p !^{r}, p \in \mathbb{N}_{0}$ behaves at infinity as $|\cdot|^{1 / r}$, cf. [53]. In fact, the interplay between the defining sequence and its associated function plays an important role in the theory of ultradistributions.

Theorem 1. Let there be given sequences of positive numbers $\left(M_{p}\right)_{p \in \mathbb{N}_{0}}$ and $\left(N_{q}\right)_{q \in \mathbb{N}_{0}}$ which satisfy (M.1) and (M.2) and $p ! \subset M_{p} N_{p}\left(p ! \prec M_{p} N_{p}\right.$, respectively). Then the following conditions are equivalent: 
1. $f \in \mathscr{S}_{M_{p}}^{N_{q}}\left(\mathbb{R}^{d}\right)\left(f \in \Sigma_{M_{p}}^{N_{q}}\left(\mathbb{R}^{d}\right)\right.$, respectively).

2. There exist constants $A, B \in \mathbb{R}^{d}, A, B>0$ (for every $A, B \in \mathbb{R}^{d}, A, B>0$ respectively), and there exist $C>0$ such that

$$
\left\|e^{M(|A x|)} \partial^{q} f(x)\right\|_{L^{\infty}} \leq C B^{q} N_{|q|}, \quad \forall p, q \in \mathbb{N}_{0}^{d} .
$$

3. There exist constants $A, B \in \mathbb{R}^{d}, A, B>0$ (for every $A, B \in \mathbb{R}^{d}, A, B>0$, respectively), and there exist $C>0$ such that

$$
\left\|x^{p} f(x)\right\|_{L^{\infty}} \leq C A^{p} M_{|p|} \quad \text { and } \quad\left\|\partial^{q} f(x)\right\|_{L^{\infty}} \leq C B^{q} N_{|q|}, \quad \forall p, q \in \mathbb{N}_{0}^{d} .
$$

4. There exist constants $A, B \in \mathbb{R}^{d}, A, B>0$ (for every $A, B \in \mathbb{R}^{d}, A, B>0$, respectively), and there exist $C>0$ such that

$$
\left\|x^{p} f(x)\right\|_{L^{\infty}} \leq C A^{p} M_{|p|} \quad \text { and } \quad\left\|\omega^{q} \hat{f}(\omega)\right\|_{L^{\infty}} \leq C B^{q} N_{|q|}, \quad \forall p, q \in \mathbb{N}_{0}^{d} .
$$

5. There exist constants $A, B \in \mathbb{R}^{d}, A, B>0$ (for every $A, B \in \mathbb{R}^{d}, A, B>0$, respectively), such that

$$
\|f(x)\|_{L^{\infty}<\infty} \text { and }\left\|\hat{f}(\omega) e^{N(|B \omega|)}\right\|_{L^{\infty}}<\infty,
$$

where $M(\cdot)$ and $N(\cdot)$ are the associated functions for the sequences $\left(M_{p}\right)_{p \in \mathbb{N}_{0}}$ and $\left(N_{q}\right)_{q \in \mathbb{N}_{0}}$ respectively.

Proof. Theorem 1 is for the first time proved in [21] and reinvented many times afterwards, see e.g. $[43,45,55,19,52]$. As an illustration, and to give a flavor of the technique, we show $1 . \Leftrightarrow 2$. For the simplicity, we observe the Gevrey sequences $M_{p}=p !^{r}$ and $N_{q}=q !^{s}, p \in \mathbb{N}_{0}, r, s>0$. that

Recall, $f \in \mathscr{S}_{M_{p}}^{N_{q}}\left(\mathbb{R}^{d}\right)=\mathscr{S}_{r}^{s}\left(\mathbb{R}^{d}\right)$ if and only if there exist constants $h, C>0$ such

$$
\sup _{x \in \mathbb{R}^{d}}\left|x^{p} \partial^{q} f(x)\right| \leq C h^{|p|+|q|} p !^{r} q !^{s}, \quad \forall p, q \in \mathbb{N}_{0}^{d} .
$$

To avoid the use of inequalities related to multi-indices we consider $d=1$. Put $F_{q}(x)=\partial^{q} f(x) /\left(h^{|q|} q^{! s}\right)$. We have

$$
\sup _{x} h^{-|p|} p !^{-r}|x|^{p}\left|F_{q}(x)\right| \leq C
$$

so that

$$
\sup _{x} h^{-|p| / r} p !^{-1}|x|^{p / r}\left|F_{q}(x)\right|^{1 / r} \leq C^{1 / r}
$$

uniformly in $p$. Therefore

$$
\sup _{x} \sum_{p \in \mathbb{N}_{0}}\left(\frac{\left(|x| h^{-1}\right)^{1 / r}}{2}\right)^{|p|} p !^{-1}\left|F_{q}(x)\right|^{1 / r} \leq C^{1 / r} \sum_{p \in \mathbb{N}_{0}} \frac{1}{2^{|p|}} .
$$


Put $A=h^{-1 / r} 2^{-1}$, and conclude that there exist constants $A, B, C>0$ such that

$$
\left|\partial^{q} f(x)\right| \leq C B^{|q|} q !^{s} e^{-A|x|^{1 / r}}, \quad \forall x \in \mathbb{R}, \quad \forall q \in \mathbb{N}_{0},
$$

which gives 2 .

Assume now that 2. holds. Put $F_{q}(x)=\partial^{q} f(x) /\left(C^{|q|} q !^{s}\right)$. Then, $\left|\partial^{q} f(x)\right| \leq$ $C^{1+|q|} q !^{s} e^{-A|x|^{1 / r}}$ for all $x \in \mathbb{R}$ implies the following chain of inclusions.

$$
\begin{aligned}
\left|F_{q}(x)\right|^{1 / r} e^{\frac{A}{r}|x|^{1 / r}}<\infty & \Rightarrow \sup _{x} \sum_{p \in \mathbb{N}_{0}} \frac{1}{p !}\left(\frac{A}{r}\right)^{p}|x|^{p / r}\left|F_{q}(x)\right|^{1 / r}<\infty \\
& \Rightarrow \sup _{x} \frac{1}{p !}\left(\frac{A}{r}\right)^{p}|x|^{p / r}\left|F_{q}(x)\right|^{1 / r}<\infty \\
& \Rightarrow \sup _{x} \frac{1}{p !^{!}}\left(\frac{A}{r}\right)^{r p}|x|^{p}\left|F_{q}(x)\right|<\infty \\
& \Rightarrow\left|x^{p} \partial^{q} f(x)\right| \leq \tilde{C}\left(\left(\frac{r}{A}\right)^{r}\right)^{|p|} C^{|q|} p !^{r} q !^{!} \\
& \Rightarrow\left|x^{p} F_{q}(x)\right| \leq \tilde{C} h^{|p|+|q|} p !^{r} q !^{s},
\end{aligned}
$$

so that $f \in \mathscr{S}_{M_{p}}^{N_{q}}(\mathbb{R})$.

The proof for $\Sigma_{M_{p}}^{N_{q}}\left(\mathbb{R}^{d}\right)$ is (almost) the same.

By the above characterization $\mathscr{F} \mathscr{S}_{M_{p}}^{N_{q}}\left(\mathbb{R}^{d}\right)=\mathscr{S}_{N_{q}}^{M_{p}}\left(\mathbb{R}^{d}\right)$. Observe that when $M_{p}$ and $N_{q}$ are chosen to be Gevrey sequences, then $\mathscr{S}_{1 / 2}^{1 / 2}\left(\mathbb{R}^{d}\right)$ is the smallest nonempty Gelfand-Shilov space invariant under the Fourier transform, see also Remark 4 below. Theorem 1 implies that $f \in \mathscr{S}_{1 / 2}^{1 / 2}\left(\mathbb{R}^{d}\right)$ if and only if $f \in \mathscr{C}^{\infty}\left(\mathbb{R}^{d}\right)$ and there exist constants $h>0, k>0$ such that

$$
\left\|f e^{h|\cdot|^{2}}\right\|_{L^{\infty}<\infty} \text { and }\left\|\hat{f} e^{k|\cdot|^{2}}\right\|_{L^{\infty}<\infty}
$$

Therefore the Hermite functions given by (1) belong to $\mathscr{S}_{1 / 2}^{1 / 2}\left(\mathbb{R}^{d}\right)$. This is an important fact when dealing with Gelfand-Shilov spaces, cf. [47, 54]. We refer to [80] for the situation below the "critical exponent" $1 / 2$.

Remark 4. Note that $\Sigma_{1 / 2}^{1 / 2}\left(\mathbb{R}^{d}\right)=\{0\}$ and $\Sigma_{s}^{s}\left(\mathbb{R}^{d}\right)$ is dense in the Schwartz space whenever $s>1 / 2$. One may consider a "fine tuning", that is the spaces $\Sigma_{M_{p}}^{N_{q}}\left(\mathbb{R}^{d}\right)$ such that

$$
\{0\}=\Sigma_{1 / 2}^{1 / 2}\left(\mathbb{R}^{d}\right) \hookrightarrow \Sigma_{M_{p}}^{N_{q}}\left(\mathbb{R}^{d}\right) \hookrightarrow \mathscr{S}_{M_{p}}^{N_{q}}\left(\mathbb{R}^{d}\right) \hookrightarrow \Sigma_{s}^{s}\left(\mathbb{R}^{d}\right), \quad s>1 / 2 .
$$

For that reason, we define sequences $\left(M_{p}\right)_{p \in \mathbb{N}_{0}}$ and $\left(N_{q}\right)_{q \in \mathbb{N}_{0}}$ by

$$
M_{p}:=p !^{\frac{1}{2}} \prod_{k=0}^{p} l_{k}=p !^{\frac{1}{2}} L_{p}, \quad p \in \mathbb{N}_{0}, \quad N_{q}:=q !^{\frac{1}{2}} \prod_{k=0}^{q} r_{k}=q !^{\frac{1}{2}} R_{q}, \quad q \in \mathbb{N}_{0}
$$


where $\left(r_{p}\right)_{p \in \mathbb{N}_{0}}$ and $\left(l_{p}\right)_{p \in \mathbb{N}_{0}}$ are sequences of positive numbers monotonically increasing to infinity such that (12) holds with the letter $s$ replaced by $r$ and $l$ respectively and which satisfy: For every $\alpha \in(0,1]$ and every $k>1$ so that $k p \in \mathbb{N}, p \in \mathbb{N}$,

$$
\max \left\{\left(\frac{r_{k p}}{r_{p}}\right)^{2},\left(\frac{l_{k p}}{l_{p}}\right)^{2}\right\} \leq k^{\alpha}, \quad p \in \mathbb{N} .
$$

Then $p$ ! $\prec M_{p} N_{p}$ and the sequences $\left(R_{p}\right)_{p \in \mathbb{N}_{0}}$ and $\left(L_{p}\right)_{p \in \mathbb{N}_{0}}\left(R_{p}=r_{1} \cdots r_{p}, L_{p}=\right.$ $l_{1} \cdots l_{p}, p \in \mathbb{N} R_{0}=1$, and $\left.L_{0}=1\right)$ satisfy conditions (M.1) and (M.2). Moreover,

$$
\max \left\{R_{p}, L_{p}\right\} \leq p !^{\alpha / 2}, p \in \mathbb{N},
$$

for every $\alpha \in(0,1]$. (For $p, q, k \in \mathbb{N}_{0}^{d}$ we have $L_{|p|}=\prod_{|k| \leq|p|} l_{|k|}$, and $R_{|q|}=$ $\prod_{|k| \leq|q|} r_{|q|}$.) Such sequences are used in the study of localization operators in the context of quasianalytic spaces in [18].

\subsection{Kernel theorem}

For the study the action of linear operators it is convenient to use their kernels. In particular, when dealing with multilinear extensions of localization operators, the kernel theorem for Glefand-Shilov spaces appears to be a crucial tool, cf. [73]. Such theorems extend the famous Schwartz kernel theorem (see $[63,86]$ ) to the spaces of ultradistributions. We refer to [58] for the proof in the case of non-quasianalytic Gelfand-Shilov spaces, and here we give a sketch of the proof for a general case from [70]. The only difference is that in quasianalytic case, the density arguments from [58] can not be used. Instead, we use arguments based on Hermite expansions in Gelfand-Shilov spaces, see [47, 50].

We need additional conditions for a sequence of positive numbers $\left(M_{p}\right)_{p \in \mathbb{N}_{0}}$ : $\{N .1\}$ There exist positive constants $A, H$ such that

$$
p !^{1 / 2} \leq A H^{p} M_{p}, \quad p \in \mathbb{N}_{0}
$$

and

(N.1) For every $H>0$ there exists $A>0$ such that

$$
p !^{1 / 2} \leq A H^{p} M_{p}, \quad p \in \mathbb{N}_{0}
$$

The conditions $\{N .1\}$ and (N.1) are taken from [49] where they are called nontriviality conditions for the spaces $\mathscr{S}_{M_{p}}^{M_{p}}\left(\mathbb{R}^{d}\right)$ and $\Sigma_{M_{p}}^{M_{p}}\left(\mathbb{R}^{d}\right)$ respectively. In fact, the following lemma is proved in [47].

Lemma 3. Let there be given a sequence of positive numbers $\left(M_{p}\right)_{p \in \mathbb{N}_{0}}$ which satisfies (M.1) and

$(M .2)^{\prime}$ There exist positive constants $A, H$ such that $M_{p+1} \leq A H^{p} M_{p}, p \in \mathbb{N}_{0}$. 
Then the following are equivalent:

1. The Hermite functions are contained in $\mathscr{S}_{M_{p}}^{N_{q}}\left(\mathbb{R}^{d}\right)\left(\right.$ in $\Sigma_{M_{p}}^{N_{q}}\left(\mathbb{R}^{d}\right)$, respectively).

2. $\left(M_{p}\right)_{p \in \mathbb{N}_{0}}$ satisfies $\{N .1\}\left(\left(M_{p}\right)_{p \in \mathbb{N}_{0}}\right.$ satisfies $(N .1)$, respectively).

3. There are positive constants $A, B$ and $H$ such that

$$
p !^{1 / 2} M_{q} \leq A B^{p+q} H^{p} M_{p+q}, \quad p, q \in \mathbb{N}_{0}
$$

(There is $B>0$ such that for every $H>0$ there exists $A>0$ such that

$$
p !^{1 / 2} M_{q} \leq A B^{p+q} H^{p} M_{p+q}, \quad p, q \in \mathbb{N}_{0}
$$

We note that the condition $(M .2)^{\prime}$ is weaker than the condition (M.2), and refer to [47, Remark 3.3] for the proof of Lemma 3.

Theorem 2. Let there be given a sequence of positive numbers $\left(M_{p}\right)_{p \in \mathbb{N}_{0}}$ which satisfies (M.1), (M.2) and $\{N .1\}$. Then the following isomorphisms hold:

1. $\mathscr{S}_{M_{p}}^{M_{p}}\left(\mathbb{R}^{d_{1}+d_{2}}\right) \cong \mathscr{S}_{M_{p}}^{M_{p}}\left(\mathbb{R}^{d_{1}}\right) \hat{\otimes} \mathscr{S}_{M_{p}}^{M_{p}}\left(\mathbb{R}^{d_{2}}\right)$

$$
\cong \mathscr{L}_{b}\left(\left(\mathscr{S}_{M_{p}}^{M_{p}}\right)^{\prime}\left(\mathbb{R}^{d_{1}}\right), \mathscr{S}_{M_{p}}^{M_{p}}\left(\mathbb{R}^{d_{2}}\right)\right)
$$

2. $\left(\mathscr{S}_{M_{p}}^{M_{p}}\right)^{\prime}\left(\mathbb{R}^{d_{1}+d_{2}}\right) \cong\left(\mathscr{S}_{M_{p}}^{M_{p}}\right)^{\prime}\left(\mathbb{R}^{d_{1}}\right) \hat{\otimes}\left(\mathscr{S}_{M_{p}}^{M_{p}}\right)^{\prime}\left(\mathbb{R}^{d_{2}}\right)$

$$
\cong \mathscr{L}_{b}\left(\mathscr{S}_{M_{p}}^{M_{p}}\left(\mathbb{R}^{d_{1}}\right),\left(\mathscr{S}_{M_{p}}^{M_{p}}\right)^{\prime}\left(\mathbb{R}^{d_{2}}\right)\right) \text {. }
$$

If the sequence $\left(M_{p}\right)_{p \in \mathbb{N}_{0}}$ satisfies (M.1), (M.2) and (N.1) instead, then the following isomorphisms hold:

3. $\Sigma_{M_{p}}^{M_{p}}\left(\mathbb{R}^{d_{1}+d_{2}}\right) \cong \Sigma_{M_{p}}^{M_{p}}\left(\mathbb{R}^{d_{1}}\right) \hat{\otimes} \Sigma_{M_{p}}^{M_{p}}\left(\mathbb{R}^{d_{2}}\right)$

$$
\cong \mathscr{L}_{b}\left(\left(\Sigma_{M_{p}}^{M_{p}}\right)^{\prime}\left(\mathbb{R}^{d_{1}}\right), \Sigma_{M_{p}}^{M_{p}}\left(\mathbb{R}^{d_{2}}\right)\right)
$$

4. $\left(\Sigma_{M_{p}}^{M_{p}}\right)^{\prime}\left(\mathbb{R}^{d_{1}+d_{2}}\right) \cong\left(\Sigma_{M_{p}}^{M_{p}}\right)^{\prime}\left(\mathbb{R}^{d_{1}}\right) \hat{\otimes}\left(\Sigma_{M_{p}}^{M_{p}}\right)^{\prime}\left(\mathbb{R}^{d_{2}}\right)$

$$
\cong \mathscr{L}_{b}\left(\Sigma_{M_{p}}^{M_{p}}\left(\mathbb{R}^{d_{1}}\right),\left(\Sigma_{M_{p}}^{M_{p}}\right)^{\prime}\left(\mathbb{R}^{d_{2}}\right)\right) .
$$

Proof. By [47, Remark 3.3] it follows that $\{N .1\}$ is equivalent to $H_{k}(x) \in \mathscr{S}_{M_{p}}^{M_{p}}\left(\mathbb{R}^{d_{1}}\right), x \in \mathbb{R}^{d_{1}}, k \in \mathbb{N}_{0}^{d_{1}}$, and $H_{l}(y) \in \mathscr{S}_{M_{p}}^{M_{p}}\left(\mathbb{R}^{d_{2}}\right), y \in \mathbb{R}^{d_{2}}, l \in \mathbb{N}_{0}^{d_{2}}$, where $H_{k}(x)$ and $H_{l}(y)$ are the Hermite functions given by (1). Now, by representation theorems from [47] and [49] and the fact that $H_{(k, l)}(x, y) \in \mathscr{S}_{M_{p}}^{M_{p}}\left(\mathbb{R}^{d_{1}+d_{2}}\right)$, $(x, y) \in \mathbb{R}^{d_{1}+d_{2}},(k, l) \in \mathbb{N}_{0}^{d_{1}+d_{2}}$, it follows that $\mathscr{S}_{M_{p}}^{M_{p}}\left(\mathbb{R}^{d_{1}}\right) \otimes \mathscr{S}_{M_{p}}^{M_{p}}\left(\mathbb{R}^{d_{2}}\right)$ is dense in $\mathscr{S}_{M_{p}}^{M_{p}}\left(\mathbb{R}^{d_{1}+d_{2}}\right)$. 
In order to obtain the isomorphism $\mathscr{S}_{M_{p}}^{M_{p}}\left(\mathbb{R}^{d_{1}+d_{2}}\right) \cong \mathscr{S}_{M_{p}}^{M_{p}}\left(\mathbb{R}^{d_{1}}\right) \hat{\otimes} \mathscr{S}_{M_{p}}^{M_{p}}\left(\mathbb{R}^{d_{2}}\right)$ it is sufficient to prove that $\mathscr{S}_{M_{p}}^{M_{p}}\left(\mathbb{R}^{d_{1}+d_{2}}\right)$ induces the $\pi=\varepsilon$ topology on the product space $\mathscr{S}_{M_{p}}^{M_{p}}\left(\mathbb{R}^{d_{1}}\right) \hat{\otimes} \mathscr{S}_{M_{p}}^{M_{p}}\left(\mathbb{R}^{d_{2}}\right)$. The topologies $\mathscr{S}_{M_{p}}^{M_{p}}\left(\mathbb{R}^{d_{1}}\right) \otimes_{\pi} \mathscr{S}_{M_{p}}^{M_{p}}\left(\mathbb{R}^{d_{2}}\right)$ and $\mathscr{S}_{M_{p}}^{M_{p}}\left(\mathbb{R}^{d_{1}}\right) \otimes_{\varepsilon} \mathscr{S}_{M_{p}}^{M_{p}}\left(\mathbb{R}^{d_{2}}\right)$ coincide since $\mathscr{S}_{M_{p}}^{M_{p}}\left(\mathbb{R}^{d}\right)$ is a nuclear space (see e.g. [49]). We refer to [86, Chapter 43] for the definition and basic facts on the $\pi$ and $\varepsilon$ topologies.

The idea of the proof is to show that the topology $\pi$ on $\mathscr{S}_{M_{p}}^{M_{p}}\left(\mathbb{R}^{d_{1}}\right) \otimes \mathscr{S}_{M_{p}}^{M_{p}}\left(\mathbb{R}^{d_{2}}\right)$ is stronger than the one induced from $\mathscr{S}_{M_{p}}^{M_{p}}\left(\mathbb{R}^{d_{1}+d_{2}}\right)$, and that the $\varepsilon$ topology on $\mathscr{S}_{M_{p}}^{M_{p}}\left(\mathbb{R}^{d_{1}}\right) \otimes \mathscr{S}_{M_{p}}^{M_{p}}\left(\mathbb{R}^{d_{2}}\right)$ is weaker than the induced one. This will imply the isomorphism

$$
\mathscr{S}_{M_{p}}^{M_{p}}\left(\mathbb{R}^{d_{1}+d_{2}}\right) \cong \mathscr{S}_{M_{p}}^{M_{p}}\left(\mathbb{R}^{d_{1}}\right) \hat{\otimes} \mathscr{S}_{M_{p}}^{M_{p}}\left(\mathbb{R}^{d_{2}}\right)
$$

That proof is quite technical, and we give here only a sketch, cf. [70] for details. For the $\pi$ topology, we use a convenient separately continuous bilinear mapping which implies the continuity of the inclusion $\mathscr{S}_{M_{p}}^{M_{p}}\left(\mathbb{R}^{d_{1}}\right) \otimes_{\pi} \mathscr{S}_{M_{p}}^{M_{p}}\left(\mathbb{R}^{d_{2}}\right) \rightarrow$ $\mathscr{S}_{M_{p}}^{M_{p}}\left(\mathbb{R}^{d_{1}+d_{2}}\right)$.

For the $\varepsilon$ topology, the proof is more technically involved. Namely, for a given equicontinuous subsets $A^{\prime} \subset \mathscr{S}_{M_{p}}^{M_{p}}\left(\mathbb{R}^{d_{1}}\right)$ and $B^{\prime} \subset \mathscr{S}_{M_{p}}^{M_{p}}\left(\mathbb{R}^{d_{2}}\right)$, we use a particularly chosen family of norms which defines a topology equivalent to the one given by Definition 3 to estimate $\left|\left\langle F_{x} \otimes \tilde{F}_{y}, \Phi(x, y)\right\rangle\right|, F_{x} \in A^{\prime}$ and $\tilde{F}_{y} \in B^{\prime}$.

Next, we use the fact that $\left(\mathscr{S}_{M_{p}}^{M_{p}}\right)^{\prime}\left(\mathbb{R}^{d_{1}}\right)$ and $\mathscr{S}_{M_{p}}^{M_{p}}\left(\mathbb{R}^{d_{2}}\right)$ are complete and that $\left(\mathscr{S}_{M_{p}}^{M_{p}}\right)^{\prime}\left(\mathbb{R}^{d_{1}}\right)$ is barreled. Moreover, $\mathscr{S}_{M_{p}}^{M_{p}}\left(\mathbb{R}^{d_{1}}\right)$ is nuclear and complete, so that [86, Proposition 50.5] implies that $\mathscr{L}_{b}\left(\left(\mathscr{S}_{M_{p}}^{M_{p}}\right)^{\prime}\left(\mathbb{R}^{d_{1}}\right), \mathscr{S}_{M_{p}}^{M_{p}}\left(\mathbb{R}^{d_{2}}\right)\right)$ is complete and that

$$
\mathscr{S}_{M_{p}}^{M_{p}}\left(\mathbb{R}^{d_{1}}\right) \hat{\otimes} \mathscr{S}_{M_{p}}^{M_{p}}\left(\mathbb{R}^{d_{2}}\right) \cong \mathscr{L}_{b}\left(\left(\mathscr{S}_{M_{p}}^{M_{p}}\right)^{\prime}\left(\mathbb{R}^{d_{1}}\right), \mathscr{S}_{M_{p}}^{M_{p}}\left(\mathbb{R}^{d_{2}}\right)\right) .
$$

This proves 1) and we leave the other claims to the reader, see also [58].

The isomorphisms in Theorem 2 2) tells us that for a given kernel-distribution $k(x, y)$ on $\mathbb{R}^{d_{1}+d_{2}}$ we may associate a continuous linear mapping $k$ of $\mathscr{S}_{M_{p}}^{M_{p}}\left(\mathbb{R}^{d_{2}}\right)$ into $\left(\mathscr{S}_{M_{p}}^{M_{p}}\right)^{\prime}\left(\mathbb{R}^{d_{1}}\right)$ as follows:

$$
\left\langle k_{\varphi}, \phi\right\rangle=\langle k(x, y), \phi(x) \varphi(y)\rangle, \quad \phi \in \mathscr{S}_{M_{p}}^{M_{p}}\left(\mathbb{R}^{d_{1}}\right),
$$

which is commonly written as $k_{\varphi}(\cdot)=\int k(\cdot, y) \varphi(y) d y$. By Theorem 2 b) it follows that the correspondence between $k(x, y)$ and $k$ is an isomorphism. Note also that the transpose ${ }^{t} k$ of the mapping $k$ is given by ${ }^{t} k_{\phi}(\cdot)=\int k(x, \cdot) \phi(x) d x$.

By the above isomorphisms we conclude that for any continuous and linear mapping between $\mathscr{S}_{M_{p}}^{M_{p}}\left(\mathbb{R}^{2 d}\right)$ to $\left(\mathscr{S}_{M_{p}}^{M_{p}}\right)^{\prime}\left(\mathbb{R}^{2 d}\right)$ one can assign a uniquely determined kernel with the above mentioned properties. We will use this fact in the proof of 
Theorem 6, and refer to [86, Chapter 52] for applications of kernel theorems in linear partial differential equations.

Remark 5. The choice of the Fourier transform invariant spaces of the form $\mathscr{S}_{M_{p}}^{M_{p}}\left(\mathbb{R}^{d}\right)$ in Theorem 2 is not accidental. We refer to [37] where it is proved that if the Hermite expansion $\sum_{k \in \mathbb{N}^{d}} a_{k} H_{k}(x)$ converges to $f\left(a_{k}\right.$ are the Hermite coefficients of $f$ ) in the sense of $\mathscr{S}_{r}^{s}\left(\mathbb{R}^{d}\right)\left(\Sigma_{r}^{s}\left(\mathbb{R}^{d}\right)\right.$, respectively), $r<s$, then it belongs to $\mathscr{S}_{r}^{r}\left(\mathbb{R}^{d}\right)\left(\Sigma_{r}^{r}\left(\mathbb{R}^{d}\right)\right.$, respectively).

\subsection{Time-frequency analysis of Gelfand-Shilov spaces}

In this section we extend the action of the time-frequency representations from Section 2 to the Gelfand-Shilov spaces and their dual spaces. To that end we observe the following modification of Definition 3.

Definition 4. Let there be given sequences of positive numbers $\left(M_{p}\right)_{p \in \mathbb{N}_{0}},\left(N_{q}\right)_{q \in \mathbb{N}_{0}}$, $\left(\tilde{M}_{p}\right)_{p \in \mathbb{N}_{0}},\left(\tilde{N}_{q}\right)_{q \in \mathbb{N}_{0}}$ which satisfy $(M .1)$ and (M.2). We define $\mathscr{S}_{M_{p}, \tilde{M}_{p}, A}^{N_{q}, \tilde{N}_{q}, B}\left(\mathbb{R}^{2 d}\right)$ to be the set of smooth functions $f \in C^{\infty}\left(\mathbb{R}^{2 d}\right)$ such that

$$
\begin{gathered}
\left\|x^{\alpha_{1}} \omega^{\alpha_{2}} \partial_{x}^{\beta_{1}} \partial_{\omega}^{\beta_{2}} f\right\|_{L^{\infty}} \leq C A^{\left|\alpha_{1}+\alpha_{2}\right|} M_{\left|\alpha_{1}\right|} \tilde{M}_{\left|\alpha_{2}\right|} B^{\left|\beta_{1}+\beta_{2}\right|} N_{\left|\beta_{1}\right|} \tilde{N}_{\left|\beta_{2}\right|}, \\
\left.\forall \alpha_{1}, \alpha_{2}, \beta_{1}, \beta_{2} \in \mathbb{N}_{0}^{d}\right\},
\end{gathered}
$$

and for some $A, B, C>0$. Gelfand-Shilov spaces are projective and inductive limits of $\mathscr{S}_{M_{p}, \tilde{M}_{p}, A}^{N_{q}, \tilde{N}_{q}, B}\left(\mathbb{R}^{2 d}\right)$ :

$$
\begin{aligned}
& \Sigma_{M_{p}, \tilde{M}_{p}}^{N_{q}, \tilde{N}_{q}}\left(\mathbb{R}^{2 d}\right):=\operatorname{proj} \lim _{A>0, B>0} \mathscr{S}_{M_{p}, N_{p}, A}^{N_{q}, \tilde{N}_{q}, B}\left(\mathbb{R}^{2 d}\right) \\
& \mathscr{S}_{M_{p}, \tilde{M}_{p}}^{N_{q}, \tilde{N}_{q}}\left(\mathbb{R}^{2 d}\right):=\text { ind } \lim _{A>0, B>0} \mathscr{S}_{M_{p}, \tilde{M}_{p}, A}^{N_{q}, \tilde{N}_{q}, B}\left(\mathbb{R}^{2 d}\right) .
\end{aligned}
$$

Clearly, the corresponding dual spaces are given by

$$
\begin{aligned}
& \left(\Sigma_{M_{p}, \tilde{M}_{p}}^{N_{q}, \tilde{N}_{q}}\right)^{\prime}\left(\mathbb{R}^{2 d}\right):=\text { ind } \lim _{A>0, B>0}\left(\mathscr{S}_{M_{p}, \tilde{M}_{p}, A}^{N_{q}, \tilde{N}_{q}, B}\right)^{\prime}\left(\mathbb{R}^{2 d}\right) ; \\
& \left(\mathscr{S}_{M_{p}, \tilde{M}_{p}}^{N_{q}, \tilde{N}_{q}}\right)^{\prime}\left(\mathbb{R}^{2 d}\right):=\operatorname{proj} \lim _{A>0, B>0}\left(\mathscr{S}_{M_{p}, \tilde{M}_{p}, A}^{N_{q}, \tilde{N}_{q}, B}\right)^{\prime}\left(\mathbb{R}^{2 d}\right) .
\end{aligned}
$$

By Theorem 1, the Fourier transform is a homeomorphism from $\Sigma_{M_{p}, \tilde{M}_{p}}^{N_{q}, \tilde{N}_{q}}\left(\mathbb{R}^{2 d}\right)$ to $\Sigma_{N_{q}, \tilde{N}_{q}}^{M_{p}, \tilde{M}_{p}}\left(\mathbb{R}^{2 d}\right)$ and, if $\mathscr{F}_{1} f$ denotes the partial Fourier transform of $f(x, \omega)$ with respect to the $x$ variable, and if $\mathscr{F}_{2} f$ denotes the partial Fourier transform of $f(x, \omega)$ with respect to the $\omega$ variable, then $\mathscr{F}_{1}$ and $\mathscr{F}_{2}$ are homeomorphisms 
from $\Sigma_{M_{p}, \tilde{M}_{p}}^{N_{q}, \tilde{N}_{q}}\left(\mathbb{R}^{2 d}\right)$ to $\Sigma_{M_{p}, \tilde{N}_{q}}^{N_{q}, \tilde{M}_{p}}\left(\mathbb{R}^{2 d}\right)$ and $\Sigma_{M_{p}, \tilde{N}_{q}}^{N_{q}, \tilde{M}_{p}}\left(\mathbb{R}^{2 d}\right)$, respectively. Similar facts hold when $\Sigma_{M_{p}, \tilde{M}_{p}}^{N_{q}, \tilde{N}_{q}}\left(\mathbb{R}^{2 d}\right)$ is replaced by $\mathscr{S}_{M_{p}, \tilde{M}_{p}}^{N_{q}, \tilde{N}_{q}}\left(\mathbb{R}^{2 d}\right),\left(\Sigma_{M_{p}, \tilde{M}_{p}}^{N_{q}, \tilde{N}_{q}}\right)^{\prime}\left(\mathbb{R}^{2 d}\right)$ or $\left(\mathscr{S}_{M_{p}, \tilde{M}_{p}}^{N_{q}, \tilde{N}_{q}}\right)^{\prime}\left(\mathbb{R}^{2 d}\right)$.

When $M_{p}=\tilde{M}_{p}$ and $N_{q}=\tilde{N}_{q}$ we use usual abbreviated notation: $\mathscr{S}_{M_{p}}^{N_{q}}\left(\mathbb{R}^{2 d}\right)=$ $\mathscr{S}_{M_{p}, \tilde{M}_{p}}^{N_{q}, \tilde{N}_{q}}\left(\mathbb{R}^{2 d}\right)$ and similarly for other spaces.

Let $\left(M_{p}\right)_{p \in \mathbb{N}_{0}}$ satisfy $(M .1),(M .2)$ and $\{N .1\}$ ((N.1), respectively). For any given $f, g \in \mathscr{S}_{M_{p}}^{M_{p}}\left(\mathbb{R}^{d}\right)\left(f, g \in \Sigma_{M_{p}}^{M_{p}}\left(\mathbb{R}^{d}\right)\right.$, respectively) the Grossmann-Royer transform of $f$ and $g$ is given by (4), i.e.

$$
R_{g} f(x, \omega)=\int e^{4 \pi i \omega(t-x)} f(2 x-t) \overline{g(t)} d t, \quad x, \omega \in \mathbb{R}^{d},
$$

and the definition can be extended to $f \in\left(\mathscr{S}_{M_{p}}^{M_{p}}\right)^{\prime}\left(\mathbb{R}^{d}\right)\left(f \in\left(\Sigma_{M_{p}}^{M_{p}}\right)^{\prime}\left(\mathbb{R}^{d}\right)\right.$, respectively) by duality.

Similarly, another time-frequency representations, the short-time Fourier transform $V_{g}$, the cross-Wigner distribution $W(f, g)$, and the cross-ambiguity function $A(f, g)$ given by (5), (6) and (7), can be extended to $f \in\left(\mathscr{S}_{M_{p}}^{M_{p}}\right)^{\prime}\left(\mathbb{R}^{d}\right)(f \in$ $\left(\Sigma_{M_{p}}^{M_{p}}\right)^{\prime}\left(\mathbb{R}^{d}\right)$, respectively) when $g \in \mathscr{S}_{M_{p}}^{M_{p}}\left(\mathbb{R}^{d}\right)\left(g \in \Sigma_{M_{p}}^{M_{p}}\left(\mathbb{R}^{d}\right)\right.$, respectively).

The following theorem and its variations is a folklore, in particular in the framework of the duality between $\mathscr{S}\left(\mathbb{R}^{2 d}\right)$ and $\mathscr{S}^{\prime}\left(\mathbb{R}^{2 d}\right)$. For Gelfand-Shilov spaces we refer to e.g. [43, $68,70,78]$.

Theorem 3. Let there be given sequences $\left(M_{p}\right)_{p \in \mathbb{N}_{0}}$ and $\left(N_{q}\right)_{q \in \mathbb{N}_{0}}$ which satisfy (M.1), (M.2) and $\{N .1\}$, and let $T F R(f, g) \in\left\{R_{g} f, V_{g} f, W(f, g), A\right\}$. If $f, g \in$ $\mathscr{S}_{M_{p}}^{N_{q}}\left(\mathbb{R}^{d}\right)$, then $T F R(f, g) \in \mathscr{S}_{M_{p}, N_{q}}^{N_{q}, M_{p}}\left(\mathbb{R}^{2 d}\right)$ and extends uniquely to a continuous map from $\left(\mathscr{S}_{M_{p}}^{N_{q}}\right)^{\prime}\left(\mathbb{R}^{d}\right) \times\left(\mathscr{S}_{N_{q}}^{M_{p}}\right)^{\prime}\left(\mathbb{R}^{d}\right)$ into $\left(\mathscr{S}_{M_{p}, N_{q}}^{N_{q}, M_{p}}\right)^{\prime}\left(\mathbb{R}^{2 d}\right)$.

Conversely, if TFR $(f, g) \in \mathscr{S}_{M_{p}, N_{q}}^{N_{q}, M_{p}}\left(\mathbb{R}^{2 d}\right)$ then $f, g \in \mathscr{S}_{M_{p}}^{N_{q}}\left(\mathbb{R}^{d}\right)$.

Let the sequences $\left(M_{p}\right)_{p \in \mathbb{N}_{0}}$ and $\left(N_{q}\right)_{q \in \mathbb{N}_{0}}$ satisfy (M.1), (M.2) and (N.1) instead. If $f, g \in \Sigma_{M_{p}}^{N_{q}}\left(\mathbb{R}^{d}\right)$, then $T F R(f, g) \in \Sigma_{M_{p}, N_{q}}^{N_{q} M_{p}}\left(\mathbb{R}^{2 d}\right)$ and extends uniquely to a continuous map from $\left(\Sigma_{M_{p}}^{N_{q}}\right)^{\prime}\left(\mathbb{R}^{d}\right) \times\left(\Sigma_{N_{q}}^{M_{p}}\right)^{\prime}\left(\mathbb{R}^{d}\right)$ into $\left(\Sigma_{M_{p}, N_{q}}^{N_{q}, M_{p}}\right)^{\prime}\left(\mathbb{R}^{2 d}\right)$.

Conversely, if $T F R(f, g) \in \Sigma_{M_{p}, N_{q}}^{N_{q}, M_{p}}\left(\mathbb{R}^{2 d}\right)$ then $f, g \in \Sigma_{M_{p}}^{N_{q}}\left(\mathbb{R}^{d}\right)$.

Proof. Since Gelfand-Shilov spaces are closed under reflections, dilations and modulations, by Lemma 1 it is enough to give the proof for the Grossmann-Royer transform, and the same conclusion holds for other time-frequency representations. But the proof is essentially the same as the proof of Proposition 16 . We recall (9):

$$
R_{g} f(x, \omega)=\left(\mathscr{F}_{2} \circ \tau^{*}\right) f \otimes \bar{g}(x, \omega), \quad x, \omega \in \mathbb{R}^{d},
$$

Since the pullback operator $\tau^{*}$ is a continuous bijection on $\mathscr{S}_{M_{p}, M_{p}}^{N_{q}, N_{q}}\left(\mathbb{R}^{2 d}\right)$, and $\mathscr{F}_{2}$ is a continuous bijection between $\mathscr{S}_{M_{p}, M_{p}}^{N_{q}, N_{q}}\left(\mathbb{R}^{2 d}\right)$ and $\mathscr{S}_{M_{p}, N_{q}}^{N_{q}, M_{p}}\left(\mathbb{R}^{2 d}\right)$, we obtain 


$$
R_{g} f \in \mathscr{S}_{M_{p}, N_{q}}^{N_{q}, M_{p}}\left(\mathbb{R}^{2 d}\right) \Leftrightarrow f \otimes \bar{g} \in \mathscr{S}_{M_{p}, M_{p}}^{N_{q}, N_{q}}\left(\mathbb{R}^{2 d}\right) \Leftrightarrow f, g \in \mathscr{S}_{M_{p}}^{N_{q}}\left(\mathbb{R}^{d}\right) .
$$

Moreover, $R_{g} f$ can be extended to a map from $\left(\mathscr{S}_{M_{p}}^{N_{q}}\right)^{\prime}\left(\mathbb{R}^{d}\right) \times\left(\mathscr{S}_{N_{q}}^{M_{p}}\right)^{\prime}\left(\mathbb{R}^{d}\right)$ into $\left(\mathscr{S}_{M_{p}, N_{q}}^{N_{1}, M_{p}}\right)^{\prime}\left(\mathbb{R}^{2 d}\right)$ by duality.

To prove that $R_{g} f \in \mathscr{S}_{M_{p}, N_{q}}^{N_{q}, M_{p}}\left(\mathbb{R}^{2 d}\right)$ when $f, g \in \mathscr{S}_{M_{p}}^{N_{q}}\left(\mathbb{R}^{d}\right)$, we could also perform direct calculations based on the following observations.

Asume that $g \in \mathscr{S}_{\tilde{M}_{p}}^{\tilde{N}_{q}}\left(\mathbb{R}^{d}\right)$ where $\left(\tilde{M}_{p}\right)_{p \in \mathbb{N}_{0}}$ and $\left(\tilde{N}_{q}\right)_{q \in \mathbb{N}_{0}}$ satisfy (M.1), (M.2), $\tilde{M}_{p} \subset M_{p}$ and $\tilde{N}_{q} \subset N_{q}$, which is a slightly more general situation. Than $f(x) \otimes g(t) \in$ $\mathscr{S}_{M_{p}, \tilde{M}_{p}}^{N_{q}, \tilde{N}_{q}}\left(\mathbb{R}^{d} \times \mathbb{R}^{d}\right)$.

Put $\varphi(x, t):=f(2 x-t) g(t)$. If we show

$$
\sup _{x, t \in \mathbb{R}^{d}}\left|x^{\alpha} t^{\beta} \varphi(x, t)\right| \leq C h^{|\alpha|+|\beta|} M_{|\alpha|} M_{|\beta|},
$$

and

$$
\sup _{x, t \in \mathbb{R}^{d}}\left|\partial_{x}^{\alpha} \partial_{t}^{\beta} \varphi(x, t)\right| \leq C k^{|\alpha|+|\beta|} N_{|\alpha|} N_{|\beta|}
$$

for some $h, k>0$, then by Theorem 1 it follows that $\varphi \in \mathscr{S}_{M_{p}, M_{p}}^{N_{q}, N_{q}}\left(\mathbb{R}^{2 d}\right)$.

The first inequality easily follows from assumptions on $f$ and $g$ and a change of variables:

$$
\sup _{x, t \in \mathbb{R}^{d}}\left|x^{\alpha} t^{\beta} f(2 x-t) g(t)\right| \leq 2^{-|\alpha|} \sup _{y, t \in \mathbb{R}^{d}}\left|(y+t)^{\alpha} t^{\beta} f(y) g(t)\right|,
$$

and (16) follows from the assumptions on $f, g$ and $\tilde{M}_{p} \subset M_{p}$. To prove (17), we use the Leibniz formula which gives

$$
\begin{aligned}
\left|\partial_{x}^{\alpha} \partial_{t}^{\beta} \varphi(x, t)\right| & =\left|\sum_{\gamma \leq \beta}\left(\begin{array}{c}
\beta \\
\gamma
\end{array}\right) \frac{1}{2^{|\alpha|+|\beta|}} \partial_{x}^{\alpha} \partial_{t}^{\gamma} f(2 x-t) \partial_{t}^{\beta-\gamma} g(t)\right| \\
& \leq C_{\alpha, \beta} \sup _{x, t \in \mathbb{R}^{d}}\left|\partial_{x}^{\alpha} \partial_{t}^{\gamma} f(2 x-t) \partial_{t}^{\beta-\gamma} g(t)\right| .
\end{aligned}
$$

Next we use $\tilde{N}_{q} \subset N_{q}$ and conditions (M.1) and (M.2) applied to the sequence $\left(N_{q}\right)$ to obtain (17). Therefore, $\varphi \in \mathscr{S}_{M_{p}, M_{p}}^{N_{q}, N_{q}}\left(\mathbb{R}^{2 d}\right)$.

Now, the partial inverse Fourier transform of $\varphi$ with respect to the second variable is continuous bijection between $\mathscr{S}_{M_{p}, M_{p}}^{N_{q}, N_{q}}\left(\mathbb{R}^{2 d}\right)$ and $\mathscr{S}_{M_{p}, N_{q}}^{N_{q}, M_{p}}\left(\mathbb{R}^{2 d}\right)$, and those spaces are closed under dilations, so that

$$
R_{g} f(x, \omega)=e^{-4 \pi i \omega x} \int e^{2 \pi i \omega(2 t)} \varphi(x, t) d t \in \mathscr{S}_{M_{p} N_{q}}^{N_{q}, M_{p}}\left(\mathbb{R}^{2 d}\right)
$$


if and only if $\varphi \in \mathscr{S}_{M_{p}, M_{p}}^{N_{q}, N_{q}}\left(\mathbb{R}^{2 d}\right)$. The extension to a map from $\left(\mathscr{S}_{M_{p}}^{N_{q}}\right)^{\prime}\left(\mathbb{R}^{d}\right) \times$ $\left(\mathscr{S}_{N_{q}}^{M_{p}}\right)^{\prime}\left(\mathbb{R}^{d}\right)$ into $\left(\mathscr{S}_{M_{p}, N_{q}}^{N_{q}, M_{p}}\right)^{\prime}\left(\mathbb{R}^{2 d}\right)$ is straightforward.

\section{Modulation Spaces}

The modulation space norms traditionally measure the joint time-frequency distribution of $f \in \mathscr{S}^{\prime}$, we refer, for instance, to [29], [38, Ch. 11-13] and the original literature quoted there for various properties and applications. It is usually sufficient to observe modulation spaces with weights which admit at most polynomial growth at infinity. However the study of ultra-distributions requires a more general approach that includes the weights of exponential or even superexponential growth, cf. $[19,80]$. Note that the general approach introduced already in [29] includes the weights of sub-exponential growth (see (19)). We refer to [30, 31] for related but even more general constructions, based on the general theory of coorbit spaces.

Weight Functions. In the sequel $v$ will always be a continuous, positive, even, submultiplicative function (submultiplicative weight), i.e., $v(0)=1, v(z)=v(-z)$, and $v\left(z_{1}+z_{2}\right) \leq v\left(z_{1}\right) v\left(z_{2}\right)$, for all $z, z_{1}, z_{2} \in \mathbb{R}^{2 d}$. Moreover, $v$ is assumed to be even in each group of coordinates, that is, $v(x, \omega)=v(-\omega, x)=v(-x, \omega)$, for any $(x, \omega) \in \mathbb{R}^{2 d}$. Submultipliciativity implies that $v(z)$ is dominated by an exponential function, i.e.

$$
\exists C, k>0 \quad \text { such that } \quad v(z) \leq C e^{k\|z\|}, \quad z \in \mathbb{R}^{2 d},
$$

and $\|z\|$ is the Euclidean norm of $z \in \mathbb{R}^{2 d}$. For example, every weight of the form

$$
v(z)=e^{s\|z\|^{b}}(1+\|z\|)^{a} \log ^{r}(e+\|z\|)
$$

for parameters $a, r, s \geq 0,0 \leq b \leq 1$ satisfies the above conditions.

For our investigation of localization operators we will mostly use the exponential weights defined by

$$
\begin{aligned}
w_{s}(z) & =w_{s}(x, \omega)=e^{s\|(x, \omega)\|}, \quad z=(x, \omega) \in \mathbb{R}^{2 d}, \\
\tau_{s}(z) & =\tau_{s}(x, \omega)=e^{s\|\omega\|} .
\end{aligned}
$$

Notice that arguing on $\mathbb{R}^{4 d}$ we may read

$$
\tau_{s}(z, \zeta)=w_{s}(\zeta) \quad z, \zeta \in \mathbb{R}^{2 d}
$$

which will be used in the sequel.

Associated to every submultiplicative weight we consider the class of so-called v-moderate weights $\mathscr{M}_{v}$. A positive, even weight function $m$ on $\mathbb{R}^{2 d}$ belongs to $\mathscr{M}_{v}$ if it satisfies the condition 


$$
m\left(z_{1}+z_{2}\right) \leq C v\left(z_{1}\right) m\left(z_{2}\right) \quad \forall z_{1}, z_{2} \in \mathbb{R}^{2 d} .
$$

We note that this definition implies that $\frac{1}{v} \lesssim m \lesssim v, m \neq 0$ everywhere, and that $1 / m \in \mathscr{M}_{v}$.

Depending on the growth of the weight function $m$, different Gelfand-Shilov classes may be chosen as fitting test function spaces for modulation spaces, see $[19,68,80]$. The widest class of weights allowing to define modulation spaces is the weight class $\mathscr{N}$. A weight function $m$ on $\mathbb{R}^{2 d}$ belongs to $\mathscr{N}$ if it is a continuous, positive function such that

$$
m(z)=o\left(e^{c z^{2}}\right), \quad \text { for }|z| \rightarrow \infty, \quad \forall c>0,
$$

with $z \in \mathbb{R}^{2 d}$. For instance, every function $m(z)=e^{s|z|^{b}}$, with $s>0$ and $0 \leq b<2$, is in $\mathscr{N}$. Thus, the weight $m$ may grow faster than exponentially at infinity. For example, the choice $m \in \mathscr{N} \backslash \mathscr{M}_{v}$ is related to the spaces of quasianalytic functions, [18]. We notice that there is a limit in enlarging the weight class for modulation spaces, imposed by Hardy's theorem: if $m(z) \geq C e^{c z^{2}}$, for some $c>\pi / 2$, then the corresponding modulation spaces are trivial [42]. We refer to [39] for a survey on the most important types of weights commonly used in time-frequency analysis.

Definition 5. Let $m \in \mathscr{N}$, and $g$ a non-zero window function in $\mathscr{S}_{1 / 2}^{1 / 2}\left(\mathbb{R}^{d}\right)$. For $1 \leq p, q \leq \infty$ the modulation space $M_{m}^{p, q}\left(\mathbb{R}^{d}\right)$ consists of all $f \in\left(\mathscr{S}_{1 / 2}^{1 / 2}\right)^{\prime}\left(\mathbb{R}^{d}\right)$ such that $V_{g} f \in L_{m}^{p, q}\left(\mathbb{R}^{2 d}\right)$ (weighted mixed-norm spaces). The norm on $M_{m}^{p, q}$ is

$$
\|f\|_{M_{m}^{p, q}}=\left\|V_{g} f\right\|_{L_{m}^{p, q}}=\left(\int_{\mathbb{R}^{d}}\left(\int_{\mathbb{R}^{d}}\left|V_{g} f(x, \omega)\right|^{p} m(x, \omega)^{p} d x\right)^{q / p} d \omega\right)^{1 / q}
$$

(with obvious changes if either $p=\infty$ or $q=\infty$ ). If $p, q<\infty$, the modulation space $M_{m}^{p, q}$ is the norm completion of $\mathscr{S}_{1 / 2}^{1 / 2}$ in the $M_{m}^{p, q}$-norm. If $p=\infty$ or $q=\infty$, then $M_{m}^{p, q}$ is the completion of $\mathscr{S}_{1 / 2}^{1 / 2}$ in the weak* topology.

In this paper we restrict ourselves to $v$-moderate weights $\mathscr{M}_{v}$. Then, for $f, g \in$ $\mathscr{S}^{(1)}\left(\mathbb{R}^{d}\right)=\Sigma_{1}^{1}\left(\mathbb{R}^{d}\right)$ the above integral is convergent thanks to Theorem 3. Namely, in view of (18), for a given $m \in \mathscr{M}_{v}$ there exist $l>0$ such that $m(x, \omega) \leq C e^{l\|(x, \omega)\|}$ and therefore

$$
\begin{aligned}
\left|\int_{\mathbb{R}^{d}}\left(\int_{\mathbb{R}^{d}}\left|V_{g} f(x, \omega)\right|^{p} m(x, \omega)^{p} d x\right)^{q / p} d \omega\right| \\
\leq C\left|\int_{\mathbb{R}^{d}}\left(\int_{\mathbb{R}^{d}}\left|V_{g} f(x, \omega)\right|^{p} e^{l p\|(x, \omega)\|} d x\right)^{q / p} d \omega\right|<\infty
\end{aligned}
$$

since by Theorems 3 and Theorem 1 we have $\left|V_{g} f(x, \omega)\right|<C e^{-s\|(x, \omega)\|}$ for every $s>0$. This implies $\mathscr{S}^{(1)} \subset M_{m}^{p, q}$. 
In particular, when $m$ is a polynomial weight of the form $m(x, \omega)=\langle x\rangle^{t}\langle\omega\rangle^{s}$ we will use the notation $M_{s, t}^{p, q}\left(\mathbb{R}^{d}\right)$ for the modulation spaces which consists of all $f \in \mathscr{S}^{\prime}\left(\mathbb{R}^{d}\right)$ such that

$$
\|f\|_{M_{s, t}^{p, q}} \equiv\left(\int_{\mathbb{R}^{d}}\left(\int_{\mathbb{R}^{d}}\left|V_{\phi} f(x, \omega)\langle x\rangle^{t}\langle\omega\rangle^{s}\right|^{p} d x\right)^{q / p} d \omega\right)^{1 / q}<\infty
$$

(with obvious interpretation of the integrals when $p=\infty$ or $q=\infty$ ).

If $p=q$, we write $M_{m}^{p}$ instead of $M_{m}^{p, p}$, and if $m(z) \equiv 1$ on $\mathbb{R}^{2 d}$, then we write $M^{p, q}$ and $M^{p}$ for $M_{m}^{p, q}$ and $M_{m}^{p, p}$, and so on.

In the next proposition we show that $M_{m}^{p, q}\left(\mathbb{R}^{d}\right)$ are Banach spaces whose definition is independent of the choice of the window $g \in M_{v}^{1} \backslash\{0\}$. In order to do so, we need the adjoint of the short-time Fourier transform.

For given window $g \in \mathscr{S}^{(1)}$ and a function $F(x, \xi) \in L_{m}^{p, q}\left(\mathbb{R}^{2 d}\right)$ we define $V_{g}^{*} F$ by

$$
\left\langle V_{g}^{*} F, f\right\rangle:=\left\langle F, V_{g} f\right\rangle,
$$

whenever the duality is well defined.

In our context, [38, Proposition 11.3.2] can be rewritten as follows.

Proposition 6. Fix $m \in \mathscr{M}_{v}$ and $g, \psi \in \mathscr{S}^{(1)}$, with $\langle g, \psi\rangle \neq 0$. Then

1. $V_{g}^{*}: L_{m}^{p, q}\left(\mathbb{R}^{2 d}\right) \rightarrow M_{m}^{p, q}\left(\mathbb{R}^{d}\right)$, and

$$
\left\|V_{g}^{*} F\right\|_{M_{m}^{p, q}} \leq C\left\|V_{\psi} g\right\|_{L_{v}^{1}}\|F\|_{L_{m}^{p, q}}
$$

2. The inversion formula holds: $I_{M_{m}^{p, q}}=\langle g, \psi\rangle^{-1} V_{g}^{*} V_{\psi}$, where $I_{M_{m}^{p, q}}$ stands for the identity operator.

3. $M_{m}^{p, q}\left(\mathbb{R}^{d}\right)$ are Banach spaces whose definition is independent on the choice of $g \in \mathscr{S}^{(1)} \backslash\{0\}$.

4. The space of admissible windows can be extended from $\mathscr{S}^{(1)}$ to $M_{v}^{1}$.

Proof. We refer to [19] for the proof which is based on the proof of [38, Proposition 11.3.2.]. Note that for 4. we need the density of $\mathscr{S}^{(1)}$ in $M_{m}^{p, q}$. This fact is not obvious, we refer to [11] for the proof. Then 4 .) follows by using standard arguments of [38, Theorem 11.3.7].

Note that this result actually implies that Definition 5 coincides with the usual definition of modulation spaces with weights of polynomial and sub-exponential growth (see, for example [13, 29, 38, 56]).

The following theorem lists some basic properties of modulation spaces. We refer to $[29,38,43,55,69,78]$ for the proof.

Theorem 4. Let $p, q, p_{j}, q_{j} \in[1, \infty]$ and $s, t, s_{j}, t_{j} \in \mathbb{R}, j=1,2$. Then:

1. $M_{s, t}^{p, q}\left(\mathbb{R}^{d}\right)$ are Banach spaces, independent of the choice of $\phi \in \mathscr{S}\left(\mathbb{R}^{d}\right) \backslash 0$;

2. if $p_{1} \leq p_{2}, q_{1} \leq q_{2}, s_{2} \leq s_{1}$ and $t_{2} \leq t_{1}$, then

$$
\mathscr{S}\left(\mathbb{R}^{d}\right) \subseteq M_{s_{1}, t_{1}}^{p_{1}, q_{1}}\left(\mathbb{R}^{d}\right) \subseteq M_{s_{2}, t_{2}}^{p_{2}, q_{2}}\left(\mathbb{R}^{d}\right) \subseteq \mathscr{S}^{\prime}\left(\mathbb{R}^{d}\right)
$$


3. $\cap_{s, t} M_{s, t}^{p, q}\left(\mathbb{R}^{d}\right)=\mathscr{S}\left(\mathbb{R}^{d}\right), \quad \cup_{s, t} M_{s, t}^{p, q}\left(\mathbb{R}^{d}\right)=\mathscr{S}^{\prime}\left(\mathbb{R}^{d}\right) ;$

4. Let $1 \leq p, q \leq \infty$, and let $w_{s}$ be given by (20). Then

$$
\begin{array}{ll}
\Sigma_{1}^{1}\left(\mathbb{R}^{d}\right)=\mathscr{S}^{(1)}\left(\mathbb{R}^{d}\right)=\bigcap_{s \geq 0} M_{w_{s}}^{p, q}\left(\mathbb{R}^{d}\right), & \left(\Sigma_{1}^{1}\right)^{\prime}\left(\mathbb{R}^{d}\right)=\bigcup_{s \geq 0} M_{1 / w_{s}}^{p, q}\left(\mathbb{R}^{d}\right), \\
\mathscr{S}_{1}^{1}\left(\mathbb{R}^{d}\right)=\mathscr{S}^{\{1\}}\left(\mathbb{R}^{d}\right)=\bigcup_{s>0} M_{w_{s}}^{p, q}\left(\mathbb{R}^{d}\right), \quad\left(\mathscr{S}_{1}^{1}\right)^{\prime}\left(\mathbb{R}^{d}\right)=\bigcap_{s>0} M_{1 / w_{s}}^{p, q}\left(\mathbb{R}^{d}\right) .
\end{array}
$$

5. For $p, q \in[1, \infty)$, the dual of $M_{s, t}^{p, q}\left(\mathbb{R}^{d}\right)$ is $M_{-s,-t}^{p^{\prime}, q^{\prime}}\left(\mathbb{R}^{d}\right)$, where $\frac{1}{p}+\frac{1}{p^{\prime}}=\frac{1}{q}+\frac{1}{q^{\prime}}=1$.

Remark 6. Alternatively, $\mathscr{S}_{1}^{1}\left(\mathbb{R}^{d}\right)$ can also be viewed as a projective limit (and its dual space as an inductive limit) of modulation spaces as follows:

$$
\mathscr{S}_{1}^{1}\left(\mathbb{R}^{d}\right)=\bigcap_{m \in \cap \mathscr{M}_{w_{s}}} M_{m}^{p, q}\left(\mathbb{R}^{d}\right), \quad\left(\mathscr{S}_{1}^{1}\right)^{\prime}\left(\mathbb{R}^{d}\right)=\bigcup_{m \in \cap \mathscr{M}_{w_{s}}} M_{1 / m}^{p, q}\left(\mathbb{R}^{d}\right),
$$

where $w_{s}$ is given by (20), see [81].

In the context of quasianalytic Gelfand-Shilov spaces, we recall (a special case of) [78, Theorem 3.9]: Let $s, t>1 / 2$ and set

$$
w_{h}(x, \omega) \equiv e^{h\left(|x|^{1 / t}+|\omega|^{1 / s}\right)}, \quad h>0, x, \omega \in \mathbb{R}^{d} .
$$

Then

$$
\begin{gathered}
\Sigma_{t}^{s}\left(\mathbb{R}^{d}\right)=\bigcap_{h>0} M_{w_{h}}^{p, q}\left(\mathbb{R}^{d}\right), \quad\left(\Sigma_{t}^{s}\right)^{\prime}\left(\mathbb{R}^{d}\right)=\bigcup_{h>0} M_{1 / w_{h}}^{p, q}\left(\mathbb{R}^{d}\right), \\
\mathscr{S}_{t}^{s}\left(\mathbb{R}^{d}\right)=\bigcup_{h>0} M_{w_{h}}^{p, q}\left(\mathbb{R}^{d}\right), \quad\left(\mathscr{S}_{t}^{s}\right)^{\prime}\left(\mathbb{R}^{d}\right)=\bigcap_{h>0} M_{1 / w_{h}}^{p, q}\left(\mathbb{R}^{d}\right) .
\end{gathered}
$$

Modulation spaces include the following well-know function spaces:

1. $M^{2}\left(\mathbb{R}^{d}\right)=L^{2}\left(\mathbb{R}^{d}\right)$, and $M_{t, 0}^{2}\left(\mathbb{R}^{d}\right)=L_{t}^{2}\left(\mathbb{R}^{d}\right)$;

2. The Feichtinger algebra: $M^{1}\left(\mathbb{R}^{d}\right)=S_{0}\left(\mathbb{R}^{d}\right)$;

3. Sobolev spaces: $M_{0, s}^{2}\left(\mathbb{R}^{d}\right)=H_{s}^{2}\left(\mathbb{R}^{d}\right)=\left\{f \mid \hat{f}(\omega)\langle\omega\rangle^{s} \in L^{2}\left(\mathbb{R}^{d}\right)\right\}$;

4. Shubin spaces: $M_{s}^{2}\left(\mathbb{R}^{d}\right)=L_{s}^{2}\left(\mathbb{R}^{d}\right) \cap H_{s}^{2}\left(\mathbb{R}^{d}\right)=Q_{s}\left(\mathbb{R}^{d}\right)$, cf. [65].

\subsection{Convolution estimates for modulation spaces}

Different theorems concerning the convolution relation between modulation spaces can be found in the literature. We recall the convolution estimates given in [13, Proposition 2.4] and in [84], which is sufficient for our purposes.

Proposition 7. Let $m \in \mathscr{M}_{v}$ defined on $\mathbb{R}^{2 d}$ and let $m_{1}(x)=m(x, 0)$ and $m_{2}(\omega)=$ $m(0, \omega)$, the restrictions to $\mathbb{R}^{d} \times\{0\}$ and $\{0\} \times \mathbb{R}^{d}$, and likewise for $v$. Let $v(\omega)>0$ be an arbitrary weight function on $\mathbb{R}^{d}$ and $1 \leq p, q, r, s, t \leq \infty$. If 


$$
\frac{1}{p}+\frac{1}{q}-1=\frac{1}{r}, \quad \text { and } \quad \frac{1}{t}+\frac{1}{t^{\prime}}=1
$$

then

$$
M_{m_{1} \otimes v}^{p, s t}\left(\mathbb{R}^{d}\right) * M_{v_{1} \otimes v_{2} v^{-1}}^{q, s t^{\prime}}\left(\mathbb{R}^{d}\right) \hookrightarrow M_{m}^{r, s}\left(\mathbb{R}^{d}\right)
$$

with norm inequality $\|f * h\|_{M_{m}^{r, s}} \lesssim\|f\|_{M_{m_{1} \otimes v}^{p, s t}}\|h\|_{M_{v_{1} \otimes v_{2} v^{-1}}^{q, s t^{\prime}}}$.

When the weights in Proposition 7 are chosen to be of the form $\langle x\rangle^{t}\langle\omega\rangle^{s}$, sharper continuity properties can be proved. For such results on multiplication and convolution in modulation spaces and in weighted Lebesgue spaces we observe the Young functional:

$$
\mathrm{R}(\mathrm{p})=\mathrm{R}\left(p_{0}, p_{1}, p_{2}\right) \equiv 2-\frac{1}{p_{0}}-\frac{1}{p_{1}}-\frac{1}{p_{2}}, \quad \mathrm{p}=\left(p_{0}, p_{1}, p_{2}\right) \in[1, \infty]^{3} .
$$

When $R(p)=0$, the Young inequality for convolution reads as

$$
\left\|f_{1} * f_{2}\right\|_{L^{p_{0}^{\prime}}} \leq\left\|f_{1}\right\|_{L^{p_{1}}}\left\|f_{2}\right\|_{L^{p_{2}}}, \quad f_{j} \in L^{p_{j}}\left(\mathbb{R}^{d}\right), \quad j=1,2 .
$$

The following theorem is an extension of the Young inequality to the case of weighted Lebesgue spaces and modulation spaces when $0 \leq R(p) \leq 1 / 2$.

Theorem 5. Let $s_{j}, t_{j} \in \mathbb{R}, p_{j}, q_{j} \in[1, \infty], j=0,1,2$. Assume that $0 \leq \mathrm{R}(\mathrm{p}) \leq 1 / 2$, $\mathrm{R}(\mathrm{q}) \leq 1$,

$$
\begin{aligned}
& 0 \quad \leq t_{j}+t_{k}, \quad j, k=0,1,2, \quad j \neq k, \\
& 0 \leq t_{0}+t_{1}+t_{2}-d \cdot \mathrm{R}(\mathrm{p}), \text { and } \\
& 0 \leq s_{0}+s_{1}+s_{2},
\end{aligned}
$$

with strict inequality in (28) when $\mathrm{R}(\mathrm{p})>0$ and $t_{j}=d \cdot \mathrm{R}(\mathrm{p})$ for some $j=0,1,2$.

Then $\left(f_{1}, f_{2}\right) \mapsto f_{1} * f_{2}$ on $C_{0}^{\infty}\left(\mathbb{R}^{d}\right)$ extends uniquely to a continuous map from

1. $L_{t_{1}}^{p_{1}}\left(\mathbb{R}^{d}\right) \times L_{t_{2}}^{p_{2}}\left(\mathbb{R}^{d}\right)$ to $L_{-t_{0}}^{p_{0}^{\prime}}\left(\mathbb{R}^{d}\right)$;

2. $M_{s_{1}, t_{1}}^{p_{1}, q_{1}}\left(\mathbb{R}^{d}\right) \times M_{s_{2}, t_{2}}^{p_{2}, q_{2}}\left(\mathbb{R}^{d}\right)$ to $M_{-s_{0},-t_{0}}^{p_{0}^{\prime}, q_{0}^{\prime}}\left(\mathbb{R}^{d}\right)$.

For the proof we refer to [84]. It is based on the detailed study of an auxiliary three-linear map over carefully chosen regions in $\mathbb{R}^{d}$ (see Subsections 3.1 and 3.2 in [84]). This result extends multiplication and convolution properties obtained in [57]. Moreover, the sufficient conditions from Theorem 5 are also necessary in the following sense.

Theorem 6. Let $p_{j}, q_{j} \in[1, \infty]$ and $s_{j}, t_{j} \in \mathbb{R}, j=0,1,2$. Assume that at least one of the following statements hold true:

1. the map $\left(f_{1}, f_{2}\right) \mapsto f_{1} * f_{2}$ on $C_{0}^{\infty}\left(\mathbb{R}^{d}\right)$ is continuously extendable to a map from $L_{t_{1}}^{p_{1}}\left(\mathbb{R}^{d}\right) \times L_{t_{2}}^{p_{2}}\left(\mathbb{R}^{d}\right)$ to $L_{-t_{0}}^{p_{0}^{\prime}}\left(\mathbb{R}^{d}\right) ;$ 
2. the map $\left(f_{1}, f_{2}\right) \mapsto f_{1} * f_{2}$ on $C_{0}^{\infty}\left(\mathbb{R}^{d}\right)$ is continuously extendable to a map from $M_{s_{1}, t_{1}}^{p_{1}, q_{1}}\left(\mathbb{R}^{d}\right) \times M_{s_{2}, t_{2}}^{p_{2}, q_{2}}\left(\mathbb{R}^{d}\right)$ to $M_{-s_{0},-t_{0}}^{p_{0}^{\prime}, q_{0}^{\prime}}\left(\mathbb{R}^{d}\right) ;$

Then (27) and (28) hold true.

\section{Localization operators}

We refer to $[13,19,18,71]$ for the continuity properties of localization operators on modulation spaces, and here we give a reformulation of such results by using the Grossmann-Royer transform instead of the cross-Wigner distribution. Furthermore, we use the Grossmann-Royer transform to define localization operators and to show that such operators are Weyl pseudodifferential operators. For our purposes the duality between $\mathscr{S}^{(1)}\left(\mathbb{R}^{d}\right)$ and $\left(\mathscr{S}^{(1)}\right)^{\prime}\left(\mathbb{R}^{d}\right)$ will suffice, and we use it here for the simplicity and for the clarity of exposition.

Definition 6. Let $f \in \mathscr{S}^{(1)}\left(\mathbb{R}^{d}\right)$. The localization operator $A_{a}^{\varphi_{1}, \varphi_{2}}$ with symbol a $\in$ $\mathscr{S}^{(1)^{\prime}}\left(\mathbb{R}^{2 d}\right)$ and windows $\varphi_{1}, \varphi_{2} \in \mathscr{S}^{(1)}\left(\mathbb{R}^{d}\right)$ is given by

$$
A_{a}^{\varphi_{1}, \varphi_{2}} f(t)=\int_{\mathbb{R}^{2 d}} a(x, \omega) R_{\check{\varphi}_{1}} f\left(\frac{x}{2}, \frac{\omega}{2}\right) R\left(\check{\varphi}_{2}(t)\right)\left(\frac{x}{2}, \frac{\omega}{2}\right) d x d \omega .
$$

In the weak sense,

$$
\begin{aligned}
\left\langle A_{a}^{\varphi_{1}, \varphi_{2}} f, g\right\rangle & =\left\langle a(x, \omega) R_{\breve{\varphi_{1}}} f\left(\frac{x}{2}, \frac{\omega}{2}\right), R_{\breve{\varphi_{2}}} g\left(\frac{x}{2}, \frac{\omega}{2}\right)\right\rangle \\
& =\left\langle a(x, \omega), R_{\breve{\varphi_{1}}} f\left(\frac{x}{2} \frac{\omega}{2}\right), R_{\check{\varphi}_{2}} g\left(\frac{x}{2}, \frac{\omega}{2}\right)\right\rangle, \quad f, g \in \mathscr{S}^{(1)}\left(\mathbb{R}^{d}\right),
\end{aligned}
$$

so that $A_{a}^{\varphi_{1}, \varphi_{2}}$ is well-defined continuous operator from $\mathscr{S}^{(1)}\left(\mathbb{R}^{d}\right)$ to $\left(\mathscr{S}^{(1)}\right)^{\prime}\left(\mathbb{R}^{d}\right)$, cf. Proposition 1.

Lemma 4. Let there be given $f \varphi_{1}, \varphi_{2} \in \mathscr{S}^{(1)}\left(\mathbb{R}^{d}\right)$ and $a \in \mathscr{S}^{(1)^{\prime}}\left(\mathbb{R}^{2 d}\right)$. Then $A_{a}^{\varphi_{1}, \varphi_{2}}$ given by (30) coincides with the usual localization operator $\tilde{A}_{a}^{\varphi_{1}, \varphi_{2}}$ defined by the short-time Fourier transform:

$$
\tilde{A}_{a}^{\varphi_{1}, \varphi_{2}} f(t)=\int_{\mathbb{R}^{2 d}} a(x, \omega) V_{\varphi_{1}} f(x, \omega) M_{\omega} T_{x} \varphi_{2}(t) d x d \omega .
$$

Proof. From Lemma 1 it follows that

$$
\begin{aligned}
\tilde{A}_{a}^{\varphi_{1}, \varphi_{2}} f(t) & =\int_{\mathbb{R}^{2 d}} a(x, \omega) e^{-\pi i x \omega} R_{\breve{\varphi}_{1}} f(x, \omega) M_{\omega} T_{x} \varphi_{2}(t) d x d \omega \\
& =\int_{\mathbb{R}^{2 d}} a(x, \omega) e^{-\pi i x \omega} R_{\breve{\varphi}_{1}} f(x, \omega) e^{\pi i x \omega} R\left(\check{\varphi}_{2}(t)\right)\left(\frac{x}{2}, \frac{\omega}{2}\right) d x d \omega \\
& =A_{a}^{\varphi_{1}, \varphi_{2}} f(t),
\end{aligned}
$$

since 
The Grossmann-Royer transform,...

$$
\begin{aligned}
R\left(\check{\varphi}_{2}(t)\right)\left(\frac{x}{2}, \frac{\omega}{2}\right) & =e^{4 \pi i \frac{\omega}{2}\left(t-\frac{x}{2}\right)} \check{\varphi}_{2}\left(2 \frac{x}{2}-t\right) \\
& =e^{-\pi i \omega x} e^{2 \pi i \omega t} \varphi_{2}(t-x) \\
& =e^{-\pi i \omega x} M_{\omega} T_{x} \varphi_{2}(t) .
\end{aligned}
$$

and the lemma is proved.

Next we show that localization operators can be represented as Weyl pseudodifferential operators.

Recall, if $\sigma \in \mathscr{S}^{(1)}\left(\mathbb{R}^{2 d}\right)$ then the Weyl pseudodifferential operator $L_{\sigma}$ is defined as the oscillatory integral:

$$
L_{\sigma} f(x)=\iint \sigma\left(\frac{x+y}{2}, \omega\right) f(y) e^{2 \pi(x-y) \cdot \omega} d y d \omega, \quad f \in \mathscr{S}^{(1)}\left(\mathbb{R}^{2 d}\right) .
$$

It extends to each $\sigma \in \mathscr{S}^{(1)^{\prime}}\left(\mathbb{R}^{2 d}\right)$, and then $L_{\sigma}$ is continuous from $\mathscr{S}^{(1)}\left(\mathbb{R}^{2 d}\right)$ to $\mathscr{S}^{(1)^{\prime}}\left(\mathbb{R}^{2 d}\right)$, and $\sigma$ is called the Weyl symbol of the pseudodifferential operator $L_{\sigma}$.

Lemma 5. Let $L_{\sigma}$ be the Weyl pseudodifferential operator with the Weyl symbol $\sigma \in \mathscr{S}^{(1)^{\prime}}\left(\mathbb{R}^{2 d}\right)$. Then we have

$$
L_{\sigma} f(t)=2^{d} \int_{\mathbb{R}^{2 d}} \sigma(x, \omega)(R f(t))(x, \omega) d x d \omega, \quad t \in \mathbb{R}^{d},
$$

or, in the weak sense

$$
\left\langle L_{\sigma} f, g\right\rangle=2^{d}\left\langle\sigma, R_{f} g\right\rangle, \quad f, g \in \mathscr{S}^{(1)}\left(\mathbb{R}^{d}\right) .
$$

Proof. The lemma is the same as [25, Proposition 40]. We give here a different proof. In fact we only use Fubini's theorem and the change of variables $2 x-y \mapsto t$ :

$$
\begin{aligned}
2^{d}\left\langle\sigma, R_{f} g\right\rangle & =2^{d} \iiint \sigma(x, \omega) e^{-4 \pi i \omega(y-x)} \bar{g}(2 x-y) f(y) d y d x d \omega \\
& =2^{d} \iint\left(\int \sigma(x, \omega) e^{4 \pi i \omega x} \bar{g}(2 x-y) d x\right) e^{-4 \pi i \omega y} f(y) d y d \omega \\
& =\iiint \sigma\left(\frac{t+y}{2}, \omega\right) e^{2 \pi i \omega(t+y)} \bar{g}(t) d t e^{-4 \pi i \omega y} f(y) d y d \omega \\
& =\iiint \sigma\left(\frac{t+y}{2}, \omega\right) e^{2 \pi i \omega(t-y)} f(y) \bar{g}(t) d t d y d \omega,
\end{aligned}
$$

therefore $2^{d}\left\langle\sigma, R_{f} g\right\rangle=\left\langle L_{\sigma} f, g\right\rangle$.

Lemmas 1 and 5 imply the well-known formula: $\left\langle L_{\sigma} f, g\right\rangle=\langle\sigma, W(g, f)\rangle$, cf. $[34,65,88]$.

Next we establish the so called Weyl connection, which shows that the set of localization operators is a subclass of the set of Weyl operators. Although the same result can be found elsewhere ([34, 6, 71]), it is given here in order to be selfcontained. The proof is based on kernel theorem for Gelfand-Shilov spaces, and direct calculation. 
Lemma 6. If $a \in \mathscr{S}^{(1)^{\prime}}\left(\mathbb{R}^{2 d}\right)$ and $\varphi_{1}, \varphi_{2} \in \mathscr{S}^{(1)}\left(\mathbb{R}^{d}\right)$, then the localization operator $A_{a}^{\varphi_{1}, \varphi_{2}}$ is Weyl pseudodifferential operator with the Weyl symbol $\sigma=2^{-d} a * R_{\varphi_{1}} \varphi_{2}$, in other words,

$$
A_{a}^{\varphi_{1}, \varphi_{2}}=2^{-d} L_{a * R_{\varphi_{1}} \varphi_{2}} .
$$

Proof. By the kernel Theorem 2 it follows that for any linear and continuous operator $T$ from $\mathscr{S}^{(1)}\left(\mathbb{R}^{2 d}\right)$ to $\mathscr{S}^{(1)^{\prime}}\left(\mathbb{R}^{2 d}\right)$, there exists a uniquely determined $k \in \mathscr{S}^{(1)^{\prime}}\left(\mathbb{R}^{2 d}\right)$ such that

$$
\langle T f, g\rangle=\langle k, g \otimes \bar{f}\rangle, \quad f, g \in \mathscr{S}^{(1)}\left(\mathbb{R}^{2 d}\right),
$$

see also $[50,71,85]$.

We first calculate the kernel of $A_{a}^{\varphi_{1}, \varphi_{2}}$, and than show that it coincides to the kernel of $L_{\sigma}$ when $\sigma=2^{-d} a * R_{\varphi_{1}} \varphi_{2}$.

From (31) and Proposition 12 . it follows:

$$
\begin{gathered}
\left\langle A_{a}^{\varphi, \phi} f, g\right\rangle=\left\langle a(x, \omega) R_{\breve{\varphi}_{1}} f\left(\frac{x}{2}, \frac{\omega}{2}\right), R_{\breve{\varphi_{2}}} g\left(\frac{x}{2}, \frac{\omega}{2}\right)\right\rangle \\
=\left\langle a(x, \omega) R_{\check{\varphi}_{1}} f\left(\frac{x}{2}, \frac{\omega}{2}\right), R_{\check{\varphi}_{2}} g\left(\frac{x}{2}, \frac{\omega}{2}\right)\right\rangle \\
=\iint_{\mathbb{R}^{2 d}} a(x, \omega)\left(\int_{\mathbb{R}^{d}} f(y) \overline{R\left(\check{\varphi_{1}}(y)\right)}\left(\frac{x}{2}, \frac{\omega}{2}\right) d y\right)\left(\int_{\mathbb{R}^{d}} \bar{g}(t) R\left(\check{\varphi_{2}}(t)\right)\left(\frac{x}{2}, \frac{\omega}{2}\right) d t\right) d x d \omega \\
=\int_{\mathbb{R}^{d}} \int_{\mathbb{R}^{d}} f(y) \bar{g}(t)\left(\iint_{\mathbb{R}^{2 d}} a(x, \omega) \overline{R\left(\check{\varphi}_{1}(y)\right)}\left(\frac{x}{2}, \frac{\omega}{2}\right) R\left(\check{\varphi}_{2}(t)\right)\left(\frac{x}{2}, \frac{\omega}{2}\right) d x d \omega\right) d t d y \\
=\langle k, g \otimes \bar{f}\rangle,
\end{gathered}
$$

where

$$
k(t, y)=\int_{\mathbb{R}^{2 d}} a(x, \omega) \overline{R(\check{\varphi}(y))}\left(\frac{x}{2}, \frac{\omega}{2}\right) R\left(\check{\varphi}_{2}(t)\right)\left(\frac{x}{2}, \frac{\omega}{2}\right) d x d \omega .
$$

Next, we calculate the kernel of $L_{a * R_{\varphi_{1}} \varphi_{2}}$. We use the covariance property of the Grossmann-Royer transform, Proposition 13 ., to obtain

$$
\begin{aligned}
& a * R_{\varphi_{1}} \varphi_{2}(p, q) \\
&=\iint_{\mathbb{R}^{2 d}} a(x, \omega) R_{\varphi_{1}} \varphi_{2}(p-x, q-\omega) d x d \omega \\
&=\iint_{\mathbb{R}^{2 d}} a(x, \omega) R_{T_{x} M_{\omega} \varphi_{1}} T_{x} M_{\omega} \varphi_{2}(p, q) d x d \omega \\
&=\iint_{\mathbb{R}^{2 d}} a(x, \omega)\left(\int_{\mathbb{R}^{d}} e^{4 \pi i q(t-p)} T_{x} M_{\omega} \varphi_{2}(2 p-t) \overline{T_{x} M_{\omega} \varphi_{1}}(t) d t\right) d x d \omega .
\end{aligned}
$$

Now, 


$$
\begin{aligned}
& \left\langle a * R_{\varphi_{1}} \varphi_{2}, R_{f} g\right\rangle \\
& =\iint_{\mathbb{R}^{4 d}} a(x, \omega)\left(\int_{\mathbb{R}^{d}} e^{4 \pi i q(t-p)} T_{x} M_{\omega} \varphi_{2}(2 p-t) \overline{T_{x} M_{\omega} \varphi_{1}}(t) d t\right) d x d \omega \\
& \times\left(\int_{\mathbb{R}^{d}} e^{-4 \pi i q(s-p)} \bar{g}(2 p-s) f(s) d s\right) d p d q \\
& =\iint_{\mathbb{R}^{3 d}} a(x, \omega)\left(\int_{\mathbb{R}^{d}} e^{4 \pi i q(t-s)} d q \int_{\mathbb{R}^{d}} T_{x} M_{\omega} \varphi_{2}(2 p-t) \overline{T_{x} M_{\omega} \varphi_{1}}(t) d t\right) \\
& \times\left(\int_{\mathbb{R}^{d}} \bar{g}(2 p-s) f(s) d s\right) d p d x d \omega \\
& =\iint_{\mathbb{R}^{3 d}} a(x, \omega) \delta(t-s) \int_{\mathbb{R}^{d}} T_{x} M_{\omega} \varphi_{2}(2 p-t) \overline{T_{x} M_{\omega} \varphi_{1}}(t) d t \\
& \times\left(\int_{\mathbb{R}^{d}} \bar{g}(2 p-s) f(s) d s\right) d p d x d \omega \\
& =\iint_{\mathbb{R}^{4 d}} a(x, \omega) T_{x} M_{\omega} \varphi_{2}(2 p-t) \overline{T_{x} M_{\omega} \varphi_{1}}(t) \bar{g}(2 p-t) f(t) d t d p d x d \omega \\
& =\iint_{\mathbb{R}^{4 d}} a(x, \omega) T_{x} M_{\omega} \varphi_{2}(2 p-t) \overline{T_{x} M_{\omega} \varphi_{1}}(t) \bar{g}(2 p-t) f(t) d t d p d x d \omega \\
& =2^{-d} \iint_{\mathbb{R}^{4 d}} a(x, \omega) T_{x} M_{\omega} \varphi_{2}(s) \overline{T_{x} M_{\omega} \varphi_{1}}(t) \bar{g}(s) f(t) d t d s d x d \omega \\
& =2^{-d} \iint_{\mathbb{R}^{4 d}} a(x, \omega) R\left(\check{\varphi}_{2}(s)\right)\left(\frac{x}{2}, \frac{\omega}{2}\right) \overline{R\left(\left(\check{\varphi}_{1}(s)\right)\right.}\left(\frac{x}{2}, \frac{\omega}{2}\right) \bar{g}(s) f(t) d t d s d x d \omega,
\end{aligned}
$$

where we deal with the oscillatory integral as we did before, and use the change of variables $2 p-t \mapsto t$.

Therefore

$$
\left\langle L_{a * R_{\varphi_{1}} \varphi_{2}} f, g\right\rangle=\langle k, g \otimes \bar{f}\rangle,
$$

where the $k$ is given by (35). By the uniqueness of the kernel we conclude that $A_{a}^{\varphi, \phi}=L_{a * R_{\varphi_{1}}} \varphi_{2}$, and the proof is finished.

Note that Lemma 6 can be proved in quasianalytic case by the same arguments. However, in this paper we do not need such an extension. Notice also that in the literature the symbol $a$ in Lemma 6 is called the anti-Wick symbol of the Weyl pseudodifferential operator $L_{\sigma}$.

Lemma 6 describes localization operators in terms of the convolution which is a smoothing operator. This implies different boundedness results of localization operators even if $a$ is an ultradistribution. In what follows we review some of these results. 


\subsection{Continuity properties}

In this subsection we recall the continuity properties from [71] obtained by using the relation between the Weyl pseudodifferential operators and localization operators, Lemma 6, and convolution results for modulation spaces from Theorem 5.

We also use sharp continuity results from [15]. There it is shown that the sufficient conditions for the continuity of the cross-Wigner distribution on modulation spaces are also necessary (in the un-weighted case). Related results can be found elsewhere, e.g. in $[74,75,71]$. In many situations such results overlap. For example, Proposition 10 in [72] coincides with certain sufficient conditions from [15, Theorem 1.1] when restricted to $\mathrm{R}(\mathrm{p})=0, t_{0}=-t_{1}$, and $t_{2}=\left|t_{0}\right|$. For our purposes it is convenient to rewrite [15, Theorem 1.1] in terms of the Grossmann-Royer transform.

Theorem 7. Let there be given $s \in \mathbb{R}$ and $p_{i}, q_{i}, p, q \in[1, \infty]$, such that

$$
p \leq p_{i}, q_{i} \leq q, \quad i=1,2
$$

and

$$
\min \left\{\frac{1}{p_{1}}+\frac{1}{p_{2}}, \frac{1}{q_{1}}+\frac{1}{q_{2}}\right\} \geq \frac{1}{p}+\frac{1}{q} .
$$

If $f, g \in \mathscr{S}\left(\mathbb{R}^{d}\right)$, then the map $(f, g) \mapsto R_{g} f$ extends to sesquilinear continuous map from $M_{|s|}^{p_{1}, q_{1}}\left(\mathbb{R}^{d}\right) \times M_{s}^{p_{2}, q_{2}}\left(\mathbb{R}^{d}\right)$ to $M_{s, 0}^{p, q}\left(\mathbb{R}^{2 d}\right)$ and

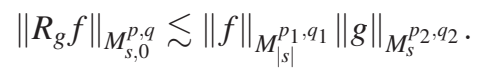

Viceversa, if there exists a constant $C>0$ such that

$$
\left\|R_{g} f\right\|_{M^{p, q}} \lesssim\|f\|_{M^{p_{1}, q_{1}}}\|g\|_{M^{p_{2}, q_{2}}} .
$$

then (36) and (37) must hold.

Proof. We refer to [15, Section 3] for the proof. It is given there in terms of the cross-Wigner distribution, which is the same as the Grossmann-Royer transform, up to the constant factor $2^{d}$.

Let $\sigma$ be the Weyl symbol of $L_{\sigma}$. By [38, Theorem 14.5.2] if $\sigma \in M^{\infty, 1}\left(\mathbb{R}^{2 d}\right)$ then $L_{\sigma}$ is bounded on $M^{p, q}\left(\mathbb{R}^{d}\right), 1 \leq p, q \leq \infty$. This result has a long history starting with the Calderon-Vaillancourt theorem on boundedness of pseudodifferential operators with smooth and bounded symbols on $L^{2}\left(\mathbb{R}^{d}\right)$, [8]. It is extended by Sjöstrand in [66] where $M^{\infty, 1}$ is used as appropriate symbol class. Sjöstrand's results were thereafter extended in [40, 38, 41, 74, 75, 76].

Theorem 8. Let the assumptions of Theorem 5 hold. If $\varphi_{j} \in M_{t_{j}}^{p_{j}}\left(\mathbb{R}^{d}\right), j=1,2$, and $a \in M_{u, v}^{\infty, r}\left(\mathbb{R}^{2 d}\right)$ where $1 \leq r \leq p_{0}, u \geq t_{0}$ and $v \geq d \mathrm{R}(\mathrm{p})$ with $v>d \mathrm{R}(\mathrm{p})$ when $\mathrm{R}(\mathrm{p})>0$, then $A_{a}^{\varphi_{1}, \varphi_{2}}$ is bounded on $M^{p, q}\left(\mathbb{R}^{d}\right)$, for all $1 \leq p, q \leq \infty$ and the operator norm satisfies the uniform estimate 


$$
\left\|A_{a}^{\varphi_{1}, \varphi_{2}}\right\|_{o p} \lesssim\|a\|_{M_{u, v}^{\infty, r}}\left\|\varphi_{1}\right\|_{M_{t_{1}}^{p_{1}}}\left\|\varphi_{2}\right\|_{M_{t_{2}}^{p_{2}}}
$$

Proof. Let $\varphi_{j} \in M_{t_{j}}^{p_{j}}\left(\mathbb{R}^{d}\right), j=1,2$. Then, by Theorem 7 it follows that $R_{\varphi_{1}} \varphi_{2} \in$ $M_{-t_{0}, 0}^{1, p_{0}^{\prime}}\left(\mathbb{R}^{2 d}\right)$. This fact, together with Theorem 5 (2) implies that

$$
a * R_{\varphi_{1}} \varphi_{2} \in M^{\tilde{p}, 1}\left(\mathbb{R}^{2 d}\right), \quad \tilde{p} \geq 2,
$$

if the involved parameters fulfill the conditions of the theorem. Concerning the Lebesgue parameters it is easy to see that $\tilde{p} \geq 2$ is equivalent to $\mathrm{R}(\mathrm{p})=\mathrm{R}(p, \infty, 1) \in$ $[0,1 / 2]$, and that $1 \leq r \leq p_{0}$ is equivalent to $\mathrm{R}(\mathrm{q})=\mathrm{R}\left(\infty, r, p_{0}^{\prime}\right) \leq 1$. It is also straightforward to check that the choice of the weight parameters $u$ and $v$ implies that $a * R_{\varphi_{1}} \varphi_{2} \in M^{\tilde{p}, 1}\left(\mathbb{R}^{2 d}\right), \tilde{p} \geq 2$.

In particular, if $\tilde{p}=\infty$ then $a * R_{\varphi_{1}} \varphi_{2} \in M^{\infty, 1}\left(\mathbb{R}^{2 d}\right)$. From [38, Theorem 14.5.2] (and Lemma 6) it follows that $A_{a}^{\varphi_{1}, \varphi_{2}}$ is bounded on $M^{p, q}\left(\mathbb{R}^{d}\right), 1 \leq p, q \leq \infty$.

The operator norm estimate also follows from [38, Theorem 14.5.2].

Remark 7. When $p_{1}=p_{2}=1, r=p_{0}=\infty$ and $t_{1}=t_{2}=-t_{0}=s \geq 0, u=-s, v=0$ we recover the celebrated Cordero-Gröchenig Theorem, [13, Theorem 3.2], in the case of polynomial weights, with the uniform estimate

$$
\left\|A_{a}^{\varphi_{1}, \varphi_{2}}\right\|_{o p} \lesssim\|a\|_{M_{-s, 0}^{\infty}}\left\|\varphi_{1}\right\|_{M_{s}^{1}}\left\|\varphi_{2}\right\|_{M_{s}^{1}}
$$

in our notation.

Another version of Theorem 8 with symbols from weighted modulation spaces can be obtained by using [38, Theorem 14.5.6] instead. We leave this to the reader as an exercise.

\subsection{Schatten-von Neumann properties}

In this subsection we recall the compactness properties from [71].

References to the proof of the following well known theorem can be found in [13].

Theorem 9. Let $\sigma$ be the Weyl symbol of $L_{\sigma}$.

1. If $\sigma \in M^{1}\left(\mathbb{R}^{2 d}\right)$ then $\left\|L_{\sigma}\right\|_{S_{1}} \lesssim\|\sigma\|_{M^{1}}$.

2. If $\sigma \in M^{p}\left(\mathbb{R}^{2 d}\right), 1 \leq p \leq 2$, then $\left\|L_{\sigma}\right\|_{S_{p}} \lesssim\|\sigma\|_{M^{p}}$.

3. If $\sigma \in M^{p, p^{\prime}}\left(\mathbb{R}^{2 d}\right), 2 \leq p \leq \infty$, then $\left\|L_{\sigma}\right\|_{S_{p}} \lesssim\|\sigma\|_{M^{p, p^{\prime}}}$.

The Schatten-von Neumann properties in the following Theorem are formulated in the spirit of [13], see also [74, 75]. Note that more general weights are considered in [76,77], leading to different type of results. We also refer to [33] for compactness properties obtained by a different approach. 
Theorem 10. Let $\mathrm{R}(\mathrm{p})$ be the Young functional given by (26), $s \geq 0$ and $t \geq d \mathrm{R}(\mathrm{p})$ with $t>d \mathrm{R}(\mathrm{p})$ when $\mathrm{R}(\mathrm{p})>0$.

1. If $1 \leq p \leq 2$ and $p \leq r \leq 2 p /(2-p)$ then the mapping $\left(a, \varphi_{1}, \varphi_{2}\right) \mapsto A_{a}^{\varphi_{1}, \varphi_{2}}$ is bounded from $M_{-s, t}^{r, q} \times M_{s}^{1} \times M_{s}^{p}$, into $S_{p}$, that is

$$
\left\|A_{a}^{\varphi_{1}, \varphi_{2}}\right\|_{S_{p}} \lesssim\|a\|_{M_{-s, t}^{r, q}}\left\|\varphi_{1}\right\|_{M_{s}^{1}}\left\|\varphi_{2}\right\|_{M_{s}^{p}}
$$

2. If $2 \leq p \leq \infty$ and $p \leq r$ then the mapping $\left(a, \varphi_{1}, \varphi_{2}\right) \mapsto A_{a}^{\varphi_{1}, \varphi_{2}}$ is bounded from $M_{-s, t}^{r, q} \times M_{s}^{1} \times M_{s}^{p^{\prime}}$, into $S_{p}$, that is

$$
\left\|A_{a}^{\varphi_{1}, \varphi_{2}}\right\|_{S_{p}} \lesssim\|a\|_{M_{-s, t}^{r, q}}\left\|\varphi_{1}\right\|_{M_{s}^{1}}\left\|\varphi_{2}\right\|_{M_{s}^{p^{\prime}}}
$$

Proof. 1. By Theorem 7 it follows that $R_{\varphi_{1}} \varphi_{2} \in M_{-t_{0}, 0}^{1, p_{w}}\left(\mathbb{R}^{2 d}\right)$, with $t_{0} \geq-s$ and $p_{w} \in[2 p /(p+2), p]$. Therefore $R_{\varphi_{1}} \varphi_{2} \in M_{s, 0}^{1, p}\left(\mathbb{R}^{2 d}\right)$.

To apply Theorem 5 we notice that for $\mathrm{R}(\mathrm{p})=\left(p^{\prime}, r, 1\right)$ the condition $\frac{1}{2} \geq \mathrm{R}(\mathrm{p}) \geq$ 0 is equivalent to $p \leq r \leq 2 p /(2-p)$, and that for $\mathrm{R}(\mathrm{p})=\left(p^{\prime}, q, p\right)$ the condition $\mathrm{R}(\mathrm{q}) \leq 1$ is equivalent to $1 \leq q$. Now, Theorem 5 implies that $a * R_{\varphi_{1}} \varphi_{2} \in M^{p}\left(\mathbb{R}^{2 d}\right)$, and the result follows from Theorem 92 .

2. By Theorem 7 it follows that $R_{\varphi_{1}} \varphi_{2} \in M_{-t_{0}, 0}^{1, p_{w}}\left(\mathbb{R}^{2 d}\right)$, with $t_{0} \geq-s$ and $p_{w} \in$ $\left[p^{\prime}, 2 p^{\prime} /\left(p^{\prime}+2\right), p\right]$. Therefore $R_{\varphi_{1}} \varphi_{2} \in M_{s, 0}^{1, p^{\prime}}\left(\mathbb{R}^{2 d}\right)$.

Now, for $2 \leq p \leq \infty, \frac{1}{2} \geq \mathrm{R}(\mathrm{p}) \geq 0$ is equivalent to $p \leq r$, and for $\mathrm{R}(\mathrm{q})=\left(p, q, p^{\prime}\right)$ the condition $\mathrm{R}(\mathrm{q}) \leq 1$ is equivalent to $1 \leq q$. The statement follows from Theorem 52 . and Theorem 93.

We remark that we corrected a typo from the formulation of [71, Theorem 3.9] also note that a particular choice: $r=p, q=\infty$ and $t=0$ recovers [13, Theorem 3.4].

In the next theorem we give some necessary conditions. The proof follow from the proofs of Theorems 4.3 and 4.4 in [13] and is therefore omitted.

Theorem 11. Let the assumptions of Theorem 5 hold and let $a \in \mathscr{S}^{\prime}\left(\mathbb{R}^{2 d}\right)$.

1. If there exists a constant $C=C(a)>0$ depending only on the symbol a such that

$$
\left\|A_{a}^{\varphi_{1}, \varphi_{2}}\right\|_{S_{\infty}} \leq C\left\|\varphi_{1}\right\|_{M_{t_{1}}^{p_{1}}}\left\|\varphi_{2}\right\|_{M_{t_{2}}^{p_{2}}}
$$

for all $\varphi_{1}, \varphi_{2} \in \mathscr{S}\left(\mathbb{R}^{d}\right)$, then $a \in M_{u, v}^{\infty, r}\left(\mathbb{R}^{2 d}\right)$ where $1 \leq r \leq p_{0}, u \geq t_{0}$ and $v \geq$ $d \mathrm{R}(\mathrm{p})$ with $v>d \mathrm{R}(\mathrm{p})$ when $\mathrm{R}(\mathrm{p})>0$.

2. If there exists a constant $C=C(a)>0$ depending only on the symbol a such that

$$
\left\|A_{a}^{\varphi_{1}, \varphi_{2}}\right\|_{S_{2}} \leq C\left\|\varphi_{1}\right\|_{M^{1}}\left\|\varphi_{2}\right\|_{M^{1}}
$$

for all $\varphi_{1}, \varphi_{2} \in \mathscr{S}\left(\mathbb{R}^{d}\right)$, then $a \in M^{2, \infty}\left(\mathbb{R}^{2 d}\right)$. 
We finish the section with results from [19] related to the weights which may have exponential growth. To that end we use the boundedness result Theorem 7 formulated in terms of such weights. The proof is a modified version of the proof of [13, Proposition 2.5] and therefore omitted.

Lemma 7. Let $w_{s}, \tau_{s}$ be the weights defined in (20) and (21). If $1 \leq p \leq \infty, s \geq 0$, $\varphi_{1} \in M_{w_{s}}^{1}\left(\mathbb{R}^{d}\right)$ and $\varphi_{2} \in M_{w_{s}}^{p}\left(\mathbb{R}^{d}\right)$, then $R_{\varphi_{1}} \varphi_{2} \in M_{\tau_{s}}^{1, p}\left(\mathbb{R}^{2 d}\right)$, and

$$
\left\|R_{\varphi_{1}} \varphi_{2}\right\|_{M_{\tau_{s}}^{1, p}} \lesssim\left\|\varphi_{1}\right\|_{M_{w_{s}}^{1}}\left\|\varphi_{2}\right\|_{M_{w_{s}}^{p}}
$$

Theorem 12. Let $A_{a}^{\varphi_{1}, \varphi_{2}}$ be the localization operator with the symbol a and windows $\varphi_{1}$ and $\varphi_{2}$.

1. If $s \geq 0, a \in M_{1 / \tau_{s}}^{\infty}\left(\mathbb{R}^{2 d}\right)$, and $\varphi_{1}, \varphi_{2} \in M_{w_{s}}^{1}\left(\mathbb{R}^{d}\right)$, then $A_{a}^{\varphi_{1}, \varphi_{2}}$ is bounded on $M^{p, q}\left(\mathbb{R}^{d}\right)$ for all $1 \leq p, q \leq \infty$, and the operator norm satisfies the uniform estimate

$$
\left\|A_{a}^{\varphi_{1}, \varphi_{2}}\right\|_{o p} \lesssim\|a\|_{M_{1 / \tau_{s}}^{\infty}}\left\|\varphi_{1}\right\|_{M_{w_{s}}^{1}}\left\|\varphi_{2}\right\|_{M_{w_{s}}^{1}} .
$$

2. If $1 \leq p \leq 2$, then the mapping $\left(a, \varphi_{1}, \varphi_{2}\right) \mapsto A_{a}^{\varphi_{1}, \varphi_{2}}$ is bounded from $M_{1 / \tau_{s}}^{p, \infty}\left(\mathbb{R}^{2 d}\right) \times$ $M_{w_{s}}^{1}\left(\mathbb{R}^{d}\right) \times M_{w_{s}}^{p}\left(\mathbb{R}^{d}\right)$ into $S_{p}$, in other words,

$$
\left\|A_{a}^{\varphi_{1}, \varphi_{2}}\right\|_{S_{p}} \lesssim\|a\|_{M_{1 / \tau_{s}}^{p, \infty}}\left\|\varphi_{1}\right\|_{M_{w_{s}}^{1}}\left\|\varphi_{2}\right\|_{M_{w_{s}}^{p}}
$$

3. If $2 \leq p \leq \infty$, then the mapping $\left(a, \varphi_{1}, \varphi_{2}\right) \mapsto A_{a}^{\varphi_{1}, \varphi_{2}}$ is bounded from $M_{1 / \tau_{s}}^{p, \infty} \times$ $M_{w_{s}}^{1} \times M_{w_{s}}^{p^{\prime}}$ into $S_{p}$, and

$$
\left\|A_{a}^{\varphi_{1}, \varphi_{2}}\right\|_{S_{p}} \lesssim\|a\|_{M_{1 / \tau_{s}}^{p, \infty}}\left\|\varphi_{1}\right\|_{M_{w_{s}}^{1}}\left\|\varphi_{2}\right\|_{M_{w_{S}}^{p^{\prime}}}
$$

Proof. 1. We use the convolution relation (25) to show that the Weyl symbol $a *$ $R_{\varphi_{1}} \varphi_{2}$ of $A_{a}^{\varphi_{1}, \varphi_{2}}$ is in $M^{\infty, 1}$. If $\varphi_{1}, \varphi_{2} \in M_{w_{s}}^{1}\left(\mathbb{R}^{d}\right)$, then by (39), we have $R_{\varphi_{1}} \varphi_{2} \in$ $M_{\tau_{s}}^{1}\left(\mathbb{R}^{2 d}\right)$. Applying Proposition 7 in the form $M_{1 / \tau_{s}}^{\infty} * M_{\tau_{s}}^{1} \subseteq M^{\infty, 1}$, we obtain $\sigma=$ $a * R_{\varphi_{1}} \varphi_{2} \in M^{\infty, 1}$. The result now follows from Theorem $9 a$ ).

Similarly, the proof of 2. and 3. is based on results of Proposition 7 and Theorem 9 , items b) - d).

For the sake of completeness, we state the necessary boundedness result, which follows by straightforward modifications of [13, Theorem 4.3].

Theorem 13. Let $a \in \mathscr{S}^{(1)^{\prime}}\left(\mathbb{R}^{2 d}\right)$ and $s \geq 0$. If there exists a constant $C=C(a)>0$ depending only on a such that

$$
\left\|A_{a}^{\varphi_{1}, \varphi_{2}}\right\|_{S_{\infty}} \leq C\left\|\varphi_{1}\right\|_{M_{w_{s}}^{1}}\left\|\varphi_{2}\right\|_{M_{w_{S}}^{1}}
$$

for all $\varphi_{1}, \varphi_{2} \in \mathscr{S}^{(1)}\left(\mathbb{R}^{d}\right)$, then $a \in M_{1 / \tau_{s}}^{\infty}$.

Note that a trace-class result for certain quasianalytic distributions (based on the heat kernel and parametrix techniques) is given in [19]. 


\section{Further extensions}

The results of previous sections can be extended in several directions. We mention here some references to product formulas, Shubin type pseudodifferential operators, multilinear localization operators, and generalizations to quasi-Banach spaces and quasianalytic spaces of test functions and their dual spaces (of Fourier ultrahyperfunctions). The list of topics and references is certainly incomplete and we leave it to the reader to accomplish it according to his/hers preferences.

The product of two localization operators can be written as a localization operator in very few cases. Exact formulas hold only in special cases, e.g. when the windows are Gaussians, [89]. Therefore, a symbolic calculus is developed in [14] where such product is written as a sum of localization operators plus a remainder term expressed in a Weyl operator form. This is done in linear case in the framework of the symbols which may have subexponential growth.

The multilinear localization operators were first introduced in [17] and their continuity properties are formulated in terms of modulation spaces. The key point is the interpretation of these operators as multilinear Kohn-Nirenberg pseudodifferential operators. The multilinear pseudodifferential operators were already studied in the context of modulation spaces in [2, 3, 4], see also a more recent contribution [51] where such approach is strengthened and applied to the bilinear and trilinear Hilbert transforms.

To deal with multilinear localization operators, instead of standard modulation spaces $M^{p, q}$ observed in [17], continuity properties in [72, 73] are formulated in terms of a modified version of modulation spaces denoted by $\mathscr{M}_{s, t}^{p, q}$, and given as follows.

By a slight abuse of the notation (as it is done in e.g. [51]), $\mathbf{f}$ denotes both the vector $\mathbf{f}=\left(f_{1}, f_{2}, \ldots, f_{n}\right)$ and the tensor product $\mathbf{f}=f_{1} \otimes f_{2} \otimes \cdots \otimes f_{n}$. This will not cause confusion, since the meaning of $\mathbf{f}$ will be clear from the context.

For example, if $t=\left(t_{1}, t_{2}, \ldots, t_{n}\right)$, and $F_{j}=F_{j}\left(t_{j}\right), t_{j} \in \mathbb{R}^{d}, j=1,2, \ldots, n$, then

$$
\prod_{j=1}^{n} F_{j}\left(t_{j}\right)=F_{1}\left(t_{1}\right) \cdot F_{2}\left(t_{2}\right) \cdots \cdots F_{n}\left(t_{n}\right)=F_{1}\left(t_{1}\right) \otimes F_{2}\left(t_{2}\right) \otimes \cdots \otimes F_{n}\left(t_{n}\right)=\mathbf{F}(t) .
$$

To give an interpretation of multilinear operators in the weak sense we note that, when $\mathbf{f}=\left(f_{1}, f_{2}, \ldots, f_{n}\right)$ and $\varphi=\left(\varphi_{1}, \varphi_{2}, \ldots, \varphi_{n}\right), f_{j}, \varphi_{j} \in \mathscr{S}^{(1)}\left(\mathbb{R}^{d}\right), j=1,2, \ldots, n$, we put

$$
R_{\varphi} \mathbf{f}(x, \omega)=\int_{\mathbb{R}^{n d}} \mathbf{f}(2 x-t) \prod_{j=1}^{n} e^{4 \pi i \omega_{j}\left(t_{j}-x_{j}\right)} \varphi_{j}\left(t_{j}\right) d t,
$$

see also (41) for the notation.

According to (42), $\mathscr{M}_{s, t}^{p, q}\left(\mathbb{R}^{n d}\right)$ denotes the the set of $\mathbf{f}=\left(f_{1}, f_{2}, \ldots, f_{n}\right), f_{j} \in$ $\mathscr{S}^{\prime}\left(\mathbb{R}^{d}\right), j=1,2, \ldots, n$, such that 


$$
\|\mathbf{f}\|_{\mathscr{M}_{s, t}^{p, q}} \equiv\left(\int_{\mathbb{R}^{n d}}\left(\int_{\mathbb{R}^{n d}}\left|V_{\varphi} \mathbf{f}(x, \omega)\langle x\rangle^{t}\langle\omega\rangle^{s}\right|^{p} d x\right)^{q / p} d \omega\right)^{1 / q}<\infty,
$$

where $\varphi=\left(\varphi_{1}, \varphi_{2}, \ldots, \varphi_{n}\right), \varphi_{j} \in \mathscr{S}\left(\mathbb{R}^{d}\right) \backslash 0, j=1,2, \ldots, n$, is a given window function.

The kernel theorem for $\mathscr{S}\left(\mathbb{R}^{d}\right)$ and $\mathscr{S}^{\prime}\left(\mathbb{R}^{d}\right)$ (see [86]) implies that there is an isomorphism between $\mathscr{M}_{s, t}^{p, q}\left(\mathbb{R}^{n d}\right)$ and $M_{s, t}^{p, q}\left(\mathbb{R}^{n d}\right)$ (which commutes with the operators from (2)). This allows us to identify $\mathbf{f} \in \mathscr{M}_{s, t}^{p, q}\left(\mathbb{R}^{n d}\right)$ with (its isomorphic image) $F \in M_{s, t}^{p, q}\left(\mathbb{R}^{n d}\right)$ (and vice versa).

Next we give multilinear version of Definition 6.

Definition 7. Let $f_{j} \in \mathscr{S}\left(\mathbb{R}^{d}\right), j=1,2, \ldots, n$, and $\mathbf{f}=\left(f_{1}, f_{2}, \ldots, f_{n}\right)$. The multilinear localization operator $A_{a}^{\varphi, \phi}$ with symbol $a \in \mathscr{S}\left(\mathbb{R}^{2 n d}\right)$ and windows

$$
\varphi=\left(\varphi_{1}, \varphi_{2}, \ldots, \varphi_{n}\right) \text { and } \phi=\left(\phi_{1}, \phi_{2}, \ldots, \phi_{n}\right), \quad \varphi_{j}, \phi_{j} \in \mathscr{S}\left(\mathbb{R}^{d}\right), \quad j=1,2, \ldots, n,
$$

is given by

$$
A_{a}^{\varphi, \phi} \mathbf{f}(t)=\int_{\mathbb{R}^{2 n d}} a(x, \omega) \prod_{j=1}^{n}\left(R_{\breve{\varphi}_{j}} f_{j}\left(\frac{x_{j}}{2}, \frac{\omega_{j}}{2}\right) R\left(\check{\phi}_{j}(t)\right)\left(\frac{x_{j}}{2}, \frac{\omega_{j}}{2}\right)\right) d x d \omega
$$

where $x_{j}, \omega_{j}, t_{j} \in \mathbb{R}^{d}, j=1,2, \ldots, n$, and $x=\left(x_{1}, x_{2}, \ldots, x_{n}\right), \omega=\left(\omega_{1}, \omega_{2} \ldots, \omega_{n}\right)$, $t=\left(t_{1}, t_{2} \ldots, t_{n}\right)$.

Let $\mathscr{R}$ denote the trace mapping that assigns to each function $F$ defined on $\mathbb{R}^{n d}$ a function defined on $\mathbb{R}^{d}$ by the formula

$$
\mathscr{R}:\left.F \mapsto F\right|_{t_{1}=t_{2}=\cdots=t_{n}}, \quad t_{j} \in \mathbb{R}^{d}, j=1,2, \ldots, n
$$

Then $\mathscr{R} A_{a}^{\varphi, \phi}$ is the multilinear operator given in [17, Definition 2.2].

The approach to multilinear localization operators related to Weyl pseudodifferential operators is given in $[72,73]$. Both Weyl and Kohn-Nirenberg correspondences are particular cases of the so-called $\tau$-pseudodifferential operators, $\tau \in[0,1]$ ( $\tau=1 / 2$ gives Weyl operators, and $\tau=0$ we reveals Kohn-Nirenberg operators). We refer to [65] for such operators, and e.g. [12, 16, 26, 83] for recent contributions in that context (see also the references given there).

The properties of such multilinear operators and their extension to GelfandShilov spaces and their dual spaces will be the subject of a separate contribution.

Furthermore, to obtain continuity properties in the framework of quasianalytic Gelfand-Shilov spaces and corresponding dual spaces analogous to those given in Section 5, another techniques should be used, cf. [18].

Finally, we mention possible extension to quasi-Banach modulation spaces, when the Lebesgue parameters $p$ and $q$ are allowed to take the values in $(0,1)$ as well. For such spaces and broad classes of pseudodifferential operators acting on them we refer to $[82,83]$. 
Acknowledgements This research is supported by MPNTR of Serbia, projects no. 174024, and DS 028 (TIFMOFUS).

\section{References}

1. Abreu, L. D., Dörfler, M., An inverse problem for localization operators, Inverse Problems 28 (11), 115001, $16 \mathrm{pp}$ (2012)

2. Bényi, Á., Gröchenig, K. H. , Heil, C. , Okoudjou, K. A., Modulation spaces and a class of bounded multilinear pseudodifferential operators, J. Operator Theory 54 (2), 387-399 (2005)

3. Bényi, Á., Okoudjou, K. A., Bilinear pseudodifferential operators on modulation spaces. J. Fourier Anal. Appl. 10 (3), 301-313 (2004)

4. Bényi, Á., Okoudjou, K. A., Modulation space estimates for multilinear pseudodifferential operators. Studia Math. 172 (2), 169-180 (2006)

5. Berezin, F. A., Wick and anti-Wick symbols of operators. Mat. Sb. (N.S.), 86(128), 578-610 (1971)

6. Boggiatto, P., Cordero, E., Gröchenig, K., Generalized Anti-Wick operators with symbols in distributional Sobolev spaces. Integral Equations Operator Theory 48, 427-442 (2004)

7. Boggiatto, P., Oliaro, A., Wong, M. W., $L^{p}$ boundedness and compactness of localization operators, J. Math. Anal. Appl. 322 (1), 193-206 (2006)

8. Calderón, A.-P., Vaillancourt, R., On the boundedness of pseudo-differential operators, J. Math. Soc. Japan 23, 374-378 (1971)

9. Cappiello, M., Gramchev, T., Rodino, L., Sub-exponential decay and uniform holomorphic extensions for semilinear pseudodifferential equations, Communications in Partial Differential Equations 35, 846-877 (2010)

10. Cappiello, M., Gramchev, T., Rodino, L., Entire extensions and exponential decay for semilinear elliptic equations, Journal d'Analyse Mathématique 111, 339-367 (2010)

11. Cordero, E., Gelfand-Shilov window classes for weighted modulation spaces. Integral Transforms Spec. Funct. 18 (11-12), 829-837 (2007)

12. Cordero, E., D'Elia L., Trapasso, S. I., Norm Estimates for $\tau$-pseudodifferential operators in Wiener amalgam and modulation spaces, arXiv:1803.07865.

13. Cordero E., Gröchenig, K., Time-frequency analysis of localization operators, Journal of Functional Analysis 205(1), 107-131 (2003)

14. Cordero E., Gröchenig, K., Symbolic calculus and Fredholm property for localization operators, J. Fourier Anal. Appl. 12 (4), 371-392 (2006)

15. Cordero E., Nicola, F., Sharp Integral Bounds for Wigner Distributions, International Mathematics Research Notices, 2016 (00), 1-29 (2016)

16. Cordero, E., Nicola F., Trapasso, S. I., Almost diagonalization of $\tau$-pseudodifferential operators with symbols in Wiener amalgam and modulation spaces, arXiv:1802.10314.

17. Cordero, E., Okoudjou, K. A., Multilinear localization operators, J. Math. Anal. Appl. 325 (2), 1103-1116 (2007)

18. Cordero, E., Pilipović, S., Rodino, L., Teofanov, N., Localization operators and exponential weights for modulation spaces, Mediterranean Journal of Mathematics 2(4), 381-394 (2005)

19. Cordero, E., Pilipović, S., Rodino, L., Teofanov, N., Quasianalytic Gelfand-Shilov spaces with application to localization operators, Rocky Mountain Journal of Mathematics, 40 (4), 1123 $1147,(2010)$

20. Chung, J., Chung, S.-Y., Kim, D., A characterization for Fourier hyperfunctions, Publ. Res. Inst. Math. Sci. 30 (2), 203-208, (1994)

21. Chung, J., Chung, S.-Y., Kim, D., Characterization of the Gelfand-Shilov spaces via Fourier transforms, Proceedings of the American Mathematical Society 124(7), 2101-2108 (1996)

22. Daubechies, I., Time-frequency localization operators: a geometric phase space approach. IEEE Trans. Inform. Theory, 34(4), 605-612 (1988) 
23. de Gosson, M., Symplectic Methods in Harmonic Analysis and in Mathematical Physics, Birkhäuser, 2011.

24. de Gosson, M., Born-Jordan Quantization, Theory and Applications, Springer (2016)

25. de Gosson, M., The Wigner Transform, World Scientific, London (2017)

26. Delgado, J., Ruzhansky, M., Wang, B., Approximation property and nuclearity on mixed-norm $L^{p}$, modulation and Wiener amalgam spaces. J. Lond. Math. Soc. 94 (2), 391-408 (2016)

27. Engliš, M., Toeplitz operators and localization operators, Trans. Amer. Math. Soc. 361 (2), 1039-1052 (2009)

28. Engliš, M., An excursion into Berezin-Toeplitz quantization and related topics, In: D. Bahns, W. Bauer, I. Witt (eds.) Oper. Theory Adv. Appl. 251, 69-115, Birkhäuser, 2016.

29. Feichtinger, H. G., Modulation spaces on locally compact abelian groups, Technical Report, University Vienna, 1983. and also in M. Krishna, R. Radha, S. Thangavelu (eds.), Wavelets and Their Applications, Allied Publishers, 99-140 (2003)

30. Feichtinger, H. G., Gröchenig, K., Banach spaces related to integrable group representations and their atomic decompositions I, J. Funct. Anal., 86(2) 307-340 (1989)

31. Feichtinger, H. G., Gröchenig, K., Banach spaces related to integrable group representations and their atomic decompositions II. Monatsh. f. Math. 108 129-148 (1989)

32. Feichtinger, H. G., Nowak, K., A first survey of Gabor multipliers, in H. Feichtinger, T. Strohmer (eds.) Advances in Gabor Analysis, Birkhäuser, 99-128 (2003)

33. Fernández, C., Galbis, A. Compactness of time-frequency localization operators on $L^{2}\left(\mathbb{R}^{d}\right)$, J. Funct. Anal. 233 (2), 335-350 (2006)

34. Folland, G. B., Harmonic Analysis in Phase Space, Princeton Univ. Press, Princeton, NJ (1989)

35. Gelfand, I. M., Shilov, G. E., On a new method in uniqueness theorems for solution of Cauchy's problem for systems of linear partial differential equations. (Russian) Dokl. Akad. Nauk SSSR (N.S.) 102, 1065-1068 (1955)

36. Gelfand, I. M., Shilov, G. E., Generalized Functions, Vol. II, III, Academic Press, New York, 1968, reprinted by the AMS (2016)

37. Gramchev, T., Lecke, A., Pilipović, S., Rodino, L., Gelfand-Shilov type spaces through Hermite expansions, in S. Pilipović, J. Toft (eds.) Pseudo-Differential Operators and Generalized Functions, Operator Theory: Advances and Applications 245, Birkhäuser, Basel, 2015.

38. Gröchenig, K. Foundations of Time-frequency analysis, Birkhäuser, Boston, 2001.

39. Gröchenig, K. H., Weight functions in time-frequency analysis. In: Rodino, L., Schulze, B.W., Wong M. W. (eds.) Pseudodifferential Operators: Partial Differential Equations and TimeFrequency Analysis, Fields Institute Comm., 52 343-366, (2007)

40. Gröchenig, K. H., Heil, C., Modulation spaces and pseudo-differential operators, Integr. Equat. Oper. Th., 34, 439-457 (1999)

41. Gröchenig, K. H. , Heil, C., Modulation spaces as symbol classes for pseudodifferential operators. In: Krishna, M., Radha, R., Thangavelu, S. (eds.) Wavelets and Their Applications, 151-170, Allied Publishers, Chennai (2003)

42. Gröchenig K., Zimmermann, G., Hardy's theorem and the short-time Fourier transform of Schwartz functions, J. London Math. Soc., 63, 205-214 (2001)

43. Gröchenig K., Zimmermann, G., Spaces of test functions via the STFT, Journal of Function Spaces and Applications 2, 25-53 (2004)

44. Grossmann, A., Parity operator and quantization of d-functions, Comm. Math. Phys. 48(3), 191-194 (1976)

45. Kamiński, A., Perišić, D., Pilipović, S., On Various Integral Transformations of Tempered Ultradistributions, Demonstratio Math., 33(3), 641-655 (2000)

46. Komatsu, H., Ultradistributions I, structure theorems and a characterization, Journal of the Faculty of Science, Section I A, Mathematics, 20, 25-105 (1973)

47. Langenbruch, M., Hermite functions and weighted spaces of generalized functions, Manuscripta Math., 119, 269-285 (2006)

48. Lerner, N., Morimoto, Y., Pravda-Starov, K., Xu, C.-J., Gelfand-Shilov smoothing properties of the radially symmetric spatially homogeneous Boltzmann equation without angular cutoff, Journal of Differential Equations 256(2), 797-831 (2014) 
49. Lozanov-Crvenković, Z., Perišić, D., Hermite expansions of elements of Gelfand-Shilov spaces in quasianalytic and non quasianalytic case, Novi Sad J. Math., 37 (2), 129-147 (2007)

50. Lozanov-Crvenković, Z., Perišić, D., Tasković, M., Gelfand-Shilov spaces, Structural and Kernel theorems, Preprint. arXiv:0706.2268v2

51. Molahajloo, S., Okoudjou K. A., Pfander, G. E., Boundedness of Multilinear Pseudodifferential Operators on Modulation Spaces, J. Fourier Anal. Appl., 22 (6), 1381-1415 (2016)

52. Nicola, F., Rodino, L., Global Pseudo-differential calculus on Euclidean spaces, PseudoDifferential Operators. Theory and Applications 4, Birkhäuser Verlag, (2010)

53. Petche, H.-J., Generalized functions and the boundary values of holomorphic functions, J. Fac. Sci. The University of Tokyo, Sec. IA, 31(2), 391-431 (1984)

54. Pilipović, S., Tempered ultradistributions, Bollettino della Unione Matematica Italiana, 7(2B), 235-251 (1988)

55. Pilipović, S., Teofanov, N., Wilson bases and ultra-modulation spaces, Math. Nachr., 242, 179-196 (2002)

56. Pilipović, S., Teofanov, N., Pseudodifferential operators on ultra-modulation spaces J. Funct. Anal., 208, 194-228 (2004)

57. Pilipović, S., Teofanov, N., Toft, J., Micro-local analysis in Fourier Lebesgue and modulation spaces, II, J. Pseudo-Differ. Oper. Appl., 1, 341-376 (2010)

58. Prangoski, B., Pseudodifferential operators of infinite order in spaces of tempered ultradistributions, Journal of Pseudo-Differential Operators and Applications, 4 (4), 495-549 (2013)

59. Prangoski, B., Laplace transform in spaces of ultradistributions, Filomat, 27(5), 747-760 (2013)

60. Ramanathan, J., Topiwala, P., Time-frequency localization via the Weyl correspondence. SIAM J. Math. Anal., 24(5), 1378-1393 (1993)

61. Rodino, L., Linear Partial Differential Operators in Gevrey Spaces, World Scientific (1993)

62. Royer, A., Wigner function as the expectation value of a parity operator. Phys. Rev. A, 15 (2), 449-450 (1977)

63. Schwartz, L., Théorie des distributions, Hermann, Paris (1950-1951)

64. Shifman, M. (editor), Felix Berezin, Life and Death of the Mastermind of Supermathematics, World Scientific, Singapore (2007)

65. Shubin, M. A., Pseudodifferential Operators and Spectral Theory, Springer-Verlag, Berlin, second edition (2001)

66. Sjöstrand, J., An algebra of pseudodifferential operators, Math. Res. Lett., 1, 185-192 (1994)

67. Slepian, D., Some comments on Fourier analysis, uncertainty and modeling, SIAM Review, 25 (3), 379-393 (1983)

68. Teofanov, N., Ultradistributions and time-frequency analysis. In: Boggiatto, P., Rodino, L., Toft, J., Wong, M. W. (eds.) Oper. Theory Adv. Appl., 164, pp. 173-191, Birkhäuser, Verlag (2006)

69. Teofanov, N., Modulation spaces, Gelfand-Shilov spaces and pseudodifferential operators, Sampl. Theory Signal Image Process, 5 (2), 225-242 (2006)

70. Teofanov, N., Gelfand-Shilov spaces and localization operators, Funct. Anal. Approx. Comput. 7 (2), 135-158 (2015)

71. Teofanov, N., Continuity and Schatten-von Neumann properties for localization operators on modulation spaces, Mediterr. J. Math. 13 (2), 745-758 (2016)

72. Teofanov, N., Bilinear Localization Operators on Modulation Spaces, Journal of Function Spaces, doi: 10.1155/2018/7560870 (2018)

73. Teofanov, N., Continuity properties of multilinear localization operators on modulation spaces, chapter in P. Boggiatto, E. Cordero, H. Feichtinger, M. de Gosson, F. Nicola, A. Oliaro, A. Tabacco (ed.), Landscapes of Time-frequency Analysis, in preparation.

74. Toft, J., Continuity properties for modulation spaces with applications to pseudo-differential calculus, I, J. Funct. Anal., 207, 399-429 (2004)

75. Toft, J., Continuity properties for modulation spaces with applications to pseudo-differential calculus, II, Ann. Global Anal. Geom., 26, 73-106 (2004) 
76. Toft, J., Continuity and Schatten properties for pseudo-differential operators on modulation spaces. In: Toft, J., Wong, M. W., Zhu, H. (eds.) Oper. Theory Adv. Appl. 172, 173-206, Birkhäuser, Verlag (2007)

77. Toft, J., Continuity and Schatten properties for Toeplitz operators on modulation spaces, In: Toft, J., Wong, M. W., Zhu, H. (eds.) Oper. Theory Adv. Appl. 172, 313-328, Birkhäuser, Verlag (2007)

78. Toft, J., The Bargmann transform on modulation and Gelfand-Shilov spaces, with applications to Toeplitz and pseudo-differential operators, J. Pseudo-Differ. Oper. Appl., 3 (2), 145-227 (2012)

79. Toft, J., Multiplication properties in Gelfand-Shilov pseudo-differential calculus, in S. Molahajloo, S. Pilipovi'c, J. Toft, M. W. Wong (eds.) Pseudo-differential operators, generalized functions and asymptotics, Operator Theory: Advances and Applications 231, 117-172, Birkhäuser/Springer, Basel (2013)

80. Toft, J., Images of function and distribution spaces under the Bargmann transform, J. PseudoDiffer. Oper. Appl. 8 (1), 83-139 (2017)

81. Toft, J., Matrix Parameterized Pseudo-differential Calculi on Modulation Spaces. In: Oberguggenberger M., Toft J., Vindas J., Wahlberg P. (eds) Generalized Functions and Fourier Analysis. Operator Theory: Advances and Applications, 260, Birkhäuser (2017)

82. Toft, J., Continuity and compactness for pseudo-differential operators with symbols in quasiBanach spaces or Hörmander classes, Anal. Appl. (Singap.) 15 (3), 353-389 (2017)

83. Toft, J., Schatten properties, nuclearity and minimality of phase shift invariant spaces, Applied and Computational Harmonic Analysis, doi.org/10.1016/j.acha.2017.04.003

84. Toft, J., Johansson, K., Pilipović, S., Teofanov, N., Sharp convolution and multiplication estimates in weighted spaces, Analysis and Applications, 13 (5), 457-480 (2015)

85. Toft, J., Khrennikov, A., Nilsson, B., Nordebo, S., Decompositions of Gelfand-Shilov kernels into kernels of similar class, J. Math. Anal. Appl. 396 (1), 315-322 (2012)

86. Treves, F., Topological Vector Spaces, Distributions and Kernels, Academic Press, New York (1967)

87. Weisz, F. Multiplier theorems for the short-time Fourier transform, Integral Equations Operator Theory 60 (1), 133-149 (2008)

88. Wong, M. W. , Weyl Transforms, Springer-Verlag (1998)

89. Wong, M. W., Wavelet transforms and localization operators, Operator Theory: Advances and Applications, 136, Birkhäuser Verlag, Basel (2002) 